\title{
NON-ARCHIMEDEAN VALUED QUASI-INVARIANT DESCENDING AT INFINITY MEASURES
}

\author{
S. V. LÜDKOVSKY
}

Received 18 May 2004 and in revised form 20 September 2005

Measures with values in non-Archimedean fields, which are quasi-invariant and descending at infinity on topological vector spaces over non-Archimedean fields, are studied in this paper. Moreover, their characteristic functionals are considered. In particular, measures having convolution properties like classical Gaussian measures are investigated in the paper. Applications of such measures to pseudodifferential operators and stochastic processes are considered. Nevertheless, it is proved that there does not exist the complete non-Archimedean analog of Gaussian measures. Theorems about either equivalence or orthogonality of measures from the considered class are proved. In addition, a pseudodifferentiability of such measures is investigated.

\section{Introduction}

This paper is devoted to new results of investigations of quasi-invariant non-Archimedean valued measures, which is becoming more important nowadays due to the development of non-Archimedean mathematical physics, particularly, quantum mechanics, quantum field theory, and theory of superstrings and supergravity $[2,3,6,10,11,15,31,32]$. On the other hand, quantum mechanics is based on measure theory and probability theory. For comparison references are given below on works, where real-valued measures on non-Archimedean spaces were studied. Stochastic approach in quantum field theory is actively used and investigated especially in recent years $[1,11,12,13]$. As it is wellknown in the theory of functions a very great role is played by continuous functions and differentiable functions.

In the classical measure theory the analog of continuity is quasi-invariance relative to shifts and actions of linear or nonlinear operators in the Banach space, differentiability of measures is the stronger condition and there is a very large theory about it in the classical case. Apart from it the non-Archimedean case was less studied. Since there are no differentiable functions from the field $\mathbb{Q}_{\|}$into $\mathbb{R}$ or in another non-Archimedean field $\mathbb{Q}_{p^{\prime}}$ with $p \neq p^{\prime}$, then instead of differentiability of measures their pseudodifferentiability is considered.

Effective ways to use quasi-invariant and pseudodifferentiable measures are given in the papers of the author $[15,16,17,18,19,20,21,22,23,24,25,26]$. I. V. Volovich was 
discussing with me the matter and was interested in results of my investigations of nonArchimedean analogs of Gaussian measures, such as measures to satisfy as many Gaussian properties as possible. He has planned to use such measures in non-Archimedean quantum field theory. The question was not so simple. He has supposed that properties with mean values, moments, projections, distributions, and convolutions of such measures can be considered analogously. But a thorough analysis has shown that not all properties can be satisfied, because in such a case the linear space would have a structure of an $\mathbb{R}$-linear space. Nevertheless, many of the properties are possible to satisfy in the nonArchimedean case also. Gaussian measures are convenient to work in the classical case, but in the non-Archimedean case they do not play such a great role.

Strictly speaking, there do not exist nontrivial Gaussian measures in the non-Archimedean case, but measures having few properties analogous to that of the Gaussian can be outlined. Supplying them with definite properties depends on a subsequent task for problems they may be useful for. Certainly if each projection $\mu_{Y}$ of a measure $\mu$ on a finite-dimensional subspace $Y$ over a field $\mathbf{K}$ is equivalent to the Haar measure $\lambda_{Y}$ on $Y$, then this is a good property. But in the classical case, as it is well-known, such property does not imply that the measure $\mu$ is Gaussian, since each measure $\nu_{Y}(d x)=f(x) \lambda_{Y}(d x)$ with $f \in L^{1}\left(Y, \lambda_{Y}, \mathbb{R}\right)$ is absolutely continuous relative to the Lebesgue measure $\lambda_{Y}$ on $Y$ and this does not imply Gaussian properties of moments or its characteristic functional $[4,9]$. The class of measures having such properties of projections is described by the Kolmogorov and Kakutani theorems. At first it is mentioned below how measures on Banach spaces can be used for construction of measures on complete ultrauniform spaces, then particular classes of quasi-invariant non-Archimedean valued measures descending at infinity are considered.

\section{Quasi-invariant descending at infinity measures}

In $[16,18]$ non-Archimedean polyhedral expansions of ultrauniform spaces were investigated and the following theorem was proved.

Theorem 2.1. Let $X$ be a complete ultrauniform space and $\mathbf{K}$ be a local field. Then there exists an irreducible normal expansion of $X$ into the limit of the inverse system $S=\left\{P_{n}, f_{n}^{m}, E\right\}$ of uniform polyhedra over $\mathbf{K}$, moreover, $\lim S$ is uniformly isomorphic with $X$, where $E$ is an ordered set, $f_{n}^{m}: P_{m} \rightarrow P_{n}$ is a continuous mapping for each $m \geq n$; particularly for the ultrametric space $(X, d)$ with the ultrametric $d$ the inverse system $S$ is the inverse sequence.

This structure theorem serves to prove the following theorem.

Theorem 2.2. Let $X$ be a complete separable ultrauniform space and let $\mathbf{K}$ be a local field. Then for each marked $b \in \mathbb{C}_{\mathrm{s}}$ there exists a nontrivial $\mathbf{F}$-valued measure $\mu$ on $X$ which is a restriction of a measure $v$ in a measure space $(Y, \mathrm{Bco}(Y), v)=\lim \left\{\left(Y_{m}, \mathrm{~B} \operatorname{co}\left(Y_{m}\right), \nu_{m}\right), \bar{f}_{n}^{m}, E\right\}$ on $X$ and each $\nu_{m}$ is quasi-invariant and pseudodifferentiable for $b \in \mathbb{C}_{s}$ relative to a dense subspace $Y_{m}^{\prime}$, where $Y_{n}:=c_{0}\left(\mathbf{K}, \alpha_{n}\right), \bar{f}_{n}^{m}: Y_{m} \rightarrow Y_{n}$ is a normal (i.e., K-simplicial nonexpanding) mapping for each $m \geq n \in E,\left.\bar{f}_{n}^{m}\right|_{P_{m}}=f_{n}^{m}$. Moreover, if $X$ is not locally compact, then the family $\mathscr{F}$ of all such $\mu$ contains a subfamily $\mathscr{G}$ of pairwise orthogonal measures with the cardinality card $(\mathscr{G})=\operatorname{card}(\mathbf{F})^{\mathrm{c}}, \mathrm{c}:=\operatorname{card}\left(\mathbb{Q}_{\mathbf{p}}\right)$. 
Proof. Choose a polyhedral expansion of $X$ in accordance with the cited above theorem. Let $\mathbb{Q}_{\mathbf{p}} \subset \mathbf{K}, s \neq p$ are prime numbers, $\mathbb{Q}_{\mathbf{s}} \subset \mathbf{F}$, where $\mathbf{F}$ is a non-Archimedean field complete relative to its uniformity. On each $X_{n}$ take a probability F-valued measure $v_{n}$ such that $\left\|X_{n} \backslash P_{n}\right\|_{v_{n}}<\epsilon_{n}, \sum_{n \in E} \epsilon_{n}<1 / 5$. In accordance with [24, 25, 26, Sections 3.5.1 and 4.2.1] (see also [23]) each $\nu_{n}$ can be chosen to be quasi-invariant and pseudodifferentiable for $b \in \mathbb{C}_{s}$ relative to a dense $\mathbf{K}$-linear subspace $Y^{\prime}{ }_{n}$, since each normal mapping $f_{n}^{m}$ has a normal extension on $Y_{m}$ supplied with the uniform polyhedra structure. Since $E$ is countable and ordered, then a family $\nu_{n}$ can be chosen by transfinite induction consistently, that is, $\bar{f}_{n}^{m}\left(\nu_{m}\right)=v_{n}$ for each $m \geq n$ in $E, \bar{f}_{n}^{m}\left(Y^{\prime}{ }_{m}\right)=Y^{\prime}{ }_{n}$. Then $X=$ $\lim \left\{P_{m}, f_{n}^{m}, E\right\} \hookrightarrow Y$. Since $\bar{f}_{n}^{m}$ are K-linear, then $\left(\bar{f}_{n}^{m}\right)^{-1}\left(\operatorname{Bco}\left(Y_{n}\right)\right) \subset \operatorname{Bco}\left(Y_{m}\right)$ for each $m \geq n \in E$. Therefore, $v$ is correctly defined on the algebra $\bigcup_{n \in E} f_{n}^{-1}\left(\operatorname{Bco}\left(Y_{n}\right)\right)$ of subsets of $Y$, where $f_{n}: X \rightarrow X_{n}$ are $\mathbf{K}$-linear continuous epimorphisms. Since $v$ is nontrivial and $\|\nu\|$ is bounded by 1 , then by the non-Archimedean analog of the Kolmogorov theorem $[21,27] \nu$ has an extension on the algebra $\operatorname{Bco}(Y)$ and hence on its completion $\operatorname{Af}(Y, v)$. Put $Y^{\prime}:=\lim \left\{Y_{m}^{\prime}, \bar{f}_{n}^{m}, E\right\}$. Then $\nu_{m}$ on $Y_{m}$ is quasi-invariant and pseudodifferentiable for $b \in \mathbb{C}_{s}$ relative to $Y^{\prime}{ }_{m}$. From $\sum_{n} \epsilon_{n}<1 / 5$ it follows that $1 \geq\|X\|_{\mu} \geq \prod_{n}\left(1-\epsilon_{n}\right)>1 / 2$, hence $\mu$ is nontrivial.

To prove the latter statement use the non-Archimedean analog of the Kakutani theorem $($ see $[24,25,26])$ for $\prod_{n} Y_{n}$ and then consider the embeddings $X \hookrightarrow Y \hookrightarrow \prod_{n} Y_{n}$ such that projection and subsequent restriction of the measure $\prod_{n} v_{n}$ on $Y$ and $X$ are nontrivial, which is possible due to the proof given above. If $\prod_{n} \nu_{n}$ and $\prod_{n} \nu_{n}^{\prime}$ are orthogonal on $\prod_{n} Y_{n}$, then they give $v$ and $v^{\prime}$ orthogonal on $X$.

\section{Definitions and notes}

A function $f: \mathbf{K} \rightarrow \mathbf{U}_{s}$ is called pseudodifferentiable of order $b$, if there exists the following integral: $\operatorname{PD}(b, f(x)):=\int_{\mathbf{K}}[(f(x)-f(y)) \times g(x, y, b)] d v(y)$. We introduce the following notation $\mathrm{PD}_{c}(b, f(x))$ for such integral by $B(\mathbf{K}, 0,1)$ instead of the entire $\mathbf{K}$. Where $g(x, y, b):=s^{(-1-b) \times \operatorname{ord}_{p}(x-y)}$ with the corresponding Haar measure $v$ with values in $\mathbf{K}_{s}$, where $\mathbf{K}_{\mathbf{s}}$ is a local field containing the field $\mathbb{Q}_{\mathbf{s}}, s$ is a prime number, $b \in \mathbb{C}_{\mathbf{s}}$ and $|x|_{\mathbf{K}}=$ $p^{-\operatorname{ord}_{p}(x)}, \mathbb{C}_{s}$ denotes the field of complex numbers with the non-Archimedean valuation extending that of $\mathbb{Q}_{s}$, and $\mathbf{U}_{s}$ is a spherically complete field with a valuation group $\Gamma_{\mathbf{U}_{s}}:=$ $\left\{|x|: 0 \neq x \in \mathbf{U}_{s}\right\}=(0, \infty) \subset \mathbb{R}$ such that $\mathbb{C}_{\mathbf{s}} \subset \mathbf{U}_{s}, 0<s$ is a prime number $[5,29,30,33]$. For each $\gamma \in(0, \infty)$ there exists $\alpha=\log _{s}(\gamma) \in \mathbb{R}, \Gamma_{\mathbf{U}_{s}}=(0, \infty)$, hence $s^{\alpha} \in \mathbf{U}_{s}$ is defined for each $\alpha \in \mathbb{R}$, where $\log _{s}(\gamma)=\ln (\gamma) / \ln (s), \ln :(0, \infty) \rightarrow \mathbb{R}$ is the natural logarithmic function such that $\ln (e)=1$. The function $s^{\alpha+i \beta}=: \xi(\alpha, \beta)$ with $\alpha$ and $\beta \in \mathbb{R}$ is defined due to the algebraic isomorphism of $\mathbb{C}_{s}$ with $\mathbb{C}$ (see [14]) in the following manner. Put $s^{\alpha+i \beta}:=s^{\alpha}\left(s^{i}\right)^{\beta}$ and choose $s^{i}$ as a marked number in $\mathbf{U}_{s}$ such that $s^{i}:=\left(\operatorname{EXP}_{s}(i)\right)^{\ln s}$, where $\operatorname{EXP}_{s}: \mathbb{C}_{s} \rightarrow \mathbb{C}_{s}^{+}$is the exponential function, $\mathbb{C}_{s}^{+}:=\left\{x \in \mathbb{C}_{s}:|x-1|_{s}<1\right\}$ (see [29, Proposition 45.6]). Therefore, $\left|\operatorname{EXP}_{s}(i)-1\right|_{s}<1$, hence $\left|\operatorname{EXP}_{s}(i)\right|_{s}=1$ and inevitably $\left|s^{i}\right|_{s}=1$. Therefore, $\left|s^{\alpha+i \beta}\right|_{s}=s^{-\alpha}$ for each $\alpha$ and $\beta \in \mathbb{R}$, where $|*|_{s}$ is the extension of the valuation from $\mathbb{Q}_{s}$ on $\mathbf{U}_{s}$, consequently, $s^{x} \in \mathbf{U}_{s}$ is defined for each $x \in \mathbb{C}_{s}$.

A quasi-invariant measure $\mu$ on $X$ is called pseudodifferentiable for $b \in \mathbb{C}_{s}$, if there exists $\operatorname{PD}(b, g(x))$ for $g(x):=\mu(-x z+S)$ for each $S \in \operatorname{Bco}(X)\|S\|_{\mu}<\infty$ and each $z \in J_{\mu}^{b}$, 
where $J_{\mu}^{b}$ is a $\mathrm{K}$-linear subspace dense in $X$. For a fixed $z \in X$ such measure is called pseudodifferentiable along $z$.

3.1. Definitions and remarks. Let $X$ be a locally $\mathbf{K}$-convex space equal to a projective $\operatorname{limit} \lim \left\{X_{j}, \phi_{l}^{j}, \Upsilon\right\}$ of Banach spaces over a local field $\mathbf{K}$ such that $X_{j}=c_{0}\left(\alpha_{j}, \mathbf{K}\right)$, where the latter space consists of vectors $x=\left(x_{k}: k \in \alpha_{j}\right), x_{k} \in \mathbf{K},\|x\|:=\sup _{k}\left|x_{k}\right|_{\mathbf{K}}<\infty$, and such that for each $\epsilon>0$ the set $\left\{k:\left|x_{k}\right|_{\mathbf{K}}>\epsilon\right\}$ is finite, $\alpha_{j}$ is a set that is convenient to consider as an ordinal due to the Kuratowski-Zorn lemma [7, 30]; $\Upsilon$ is an ordered set, $\phi_{l}^{j}$ : $X_{j} \rightarrow X_{l}$ is a K-linear continuous mapping for each $j \geq l \in \Upsilon, \phi_{j}: X \rightarrow X_{j}$ is a projection on $X_{j}, \phi_{l} \circ \phi_{l}^{j}=\phi_{j}$ for each $j \geq l \in \Upsilon$, and $\phi_{k}^{l} \circ \phi_{l}^{j}=\phi_{k}^{j}$ for each $j \geq l \geq k$ in $\Upsilon$. Consider also a locally $\mathbb{R}$-convex space that is a projective limit $Y=\lim \left\{l_{2}\left(\alpha_{j}, \mathbb{R}\right), \psi_{l}^{j}, \Upsilon\right\}$, where $l_{2}\left(\alpha_{j}, \mathbb{R}\right)$ is the real Hilbert space of the topological weight $w\left(l_{2}\left(\alpha_{j}, \mathbb{R}\right)\right)=\operatorname{card}\left(\alpha_{j}\right) \aleph_{0}$. Suppose $B$ is a symmetric nonnegative definite (bilinear) nonzero functional $B: Y^{2} \rightarrow \mathbb{R}$.

Consider a non-Archimedean field $\mathbf{F}$ such that $\mathbf{K}_{\mathbf{s}} \subset \mathbf{F}$ and with the valuation group $\Gamma_{\mathbf{F}}=(0, \infty) \subset \mathbb{R}$ and $\mathbf{F}$ is complete relative to its uniformity (see $\left.[5,8]\right)$. Then a measure $\mu=\mu_{q, B, \gamma}$ on $X$ with values in $\mathbf{K}_{\mathbf{s}}$ is called a $q$-Gaussian measure if its characteristic functional $\hat{\mu}$ with values in $\mathbf{F}$ has the form

$$
\widehat{\mu}(z)=s^{\left[B\left(v_{q}^{s}(z), v_{q}^{s}(z)\right)\right]} \chi_{\gamma}(z)
$$

on a dense $\mathbf{K}$-linear subspace $\mathrm{D}_{q, B, X}$ in $X^{*}$ of all continuous $\mathbf{K}$-linear functionals $z: X \rightarrow \mathbf{K}$ of the form $z(x)=z_{j}\left(\phi_{j}(x)\right)$ for each $x \in X$ with $v_{q}^{s}(z) \in \mathrm{D}_{B, Y}$, where $B$ is a nonnegative definite bilinear $\mathbb{R}$-valued symmetric functional on a dense $\mathbb{R}$-linear subspace $\mathrm{D}_{B, Y}$ in $Y^{*}, B: \mathrm{D}_{B, Y}^{2} \rightarrow \mathbb{R}, j \in \Upsilon$ may depend on $z, z_{j}: X_{j} \rightarrow \mathbf{K}$ is a continuous $\mathbf{K}$-linear functional such that $z_{j}=\sum_{k \in \alpha_{j}} e_{j}^{k} z_{k, j}$ is a countable convergent series such that $z_{k, j} \in \mathbf{K}$, $e_{j}^{k}$ is a continuous $\mathbf{K}$-linear functional on $X_{j}$ such that $e_{j}^{k}\left(e_{l, j}\right)=\delta_{l}^{k}$ is the Kronecker delta symbol, $e_{l, j}$ is the standard orthonormal (in the non-Archimedean sense) basis in $c_{0}\left(\alpha_{j}, \mathbf{K}\right), v_{q}^{s}(z)=v_{q}^{s}\left(z_{j}\right):=\left\{\left|s^{q \operatorname{ord}_{p}\left(z_{k, j}\right) / 2}\right|_{s}: k \in \alpha_{j}\right\}$. It is supposed that $z$ is such that $v_{q}^{s}(z) \in l_{2}\left(\alpha_{j}, \mathbb{R}\right)$, where $q$ is a positive constant, $\chi_{\gamma}(z): X \rightarrow \mathbf{T}_{\mathbf{s}}$ is a continuous character such that $\chi_{\gamma}(z)=\chi(z(\gamma)), \gamma \in X, \chi: \mathbf{K} \rightarrow \mathbf{T}_{\mathbf{s}}$ is a nontrivial character of $\mathbf{K}$ as an additive group (see [30] and $[24,25,26$, Section 2.5]).

Proposition 3.1. A q-Gaussian quasimeasure on an algebra of cylindrical subsets $\bigcup_{j} \pi_{j}^{-1}\left(\mathscr{R}_{j}\right)$, where $X_{j}$ are finite-dimensional over $\mathbf{K}$ subspaces in $X$, is a measure on a covering ring $\mathscr{R}$ of subsets of $X$ (see [24, 25, 26, Section 2.36]). Moreover, a correlation operator $B$ is of class $L_{1}$, that is, $\operatorname{Tr}(B)<\infty$, if and only if each finite-dimensional over $\mathbf{K}$ projection of $\mu$ is a q-Gaussian measure (see Section 3.1).

Proof. From Section 3.1 it follows that each one-dimensional over $\mathbf{K}$ projection $\mu_{x \mathbf{K}}$ of a measure $\mu$ satisfies $[24,25,26$, conditions $2.1(\mathrm{i})-(\mathrm{iii})]$ the covering ring $\mathrm{Bco}(\mathbf{K})$, where $0 \neq x=e_{k, l} \in X_{l}$. Therefore, $\mu$ is defined and finite additive on a cylindrical algebra:

$$
\mathrm{U}:=\bigcup_{k_{1}, \ldots, k_{n} ; l} \phi_{l}^{-1}\left[\left(\phi_{k_{1}, \ldots, k_{n}}^{l}\right)^{-1}\left(\operatorname{Bco}\left(\operatorname{span}_{\mathbf{K}}\left\{e_{k_{1}, l}, \ldots, e_{k_{n}, l}\right\}\right)\right)\right]
$$


where $\phi_{k_{1}, \ldots, k_{n}}^{l}: X_{l} \rightarrow \operatorname{span}_{\mathbf{K}}\left(e_{k_{1}, l}, \ldots, e_{k_{n}, l}\right)$ is a projection. This means that $\mu$ is a bounded quasimeasure on $\mathrm{U}$. Since $\hat{\mu}(0)=1$, then $\mu(X)=1$. The characteristic functional $\hat{\mu}$ satisfies $[24,25,26$, Conditions 2.5.(3,5)]. In view of the non-Archimedean analog of the Bochner-Kolmogorov theorem [24, 25, 26, Section 2.21 and Theorem 2.37] $\mu$ has an extension to a probability measure on a covering ring $\mathscr{R}$ of subsets of $X$ containing $\mathrm{U}$.

Suppose that $B$ is of class $L_{1}$. Then $B\left(v_{q}(z), v_{q}(z)\right)$ and hence $\widehat{\mu}(z)$ is correctly defined for each $z \in \mathrm{D}_{q, B, X}$. The set $\mathrm{D}_{q, B, X}$ of functionals $z$ on $X$ from Section 3.1 separates points of $X$. From Section 3.1 it follows that $\hat{\mu}(y)$ is continuous. Consider a diagonal compact operator $T$ in the standard orthonormal base, $T e_{k, l}=a_{k, l} e_{k, l}, \lim _{k+l \rightarrow \infty} a_{k, l}=0$. Since $B$ is continuous, then the correlation operator $E$ corresponding to $B$ is a bounded $\mathrm{K}$-linear operator on $Y,\|E\|<\infty$. For each $\epsilon>0$ there exist $\delta>0$ and $T$ such that $\max (1$, $\|E\|) \delta<\epsilon$ and $\left|a_{k, l}\right|<\delta$ for each $k+l>\mathbb{N}$, where $\mathbb{N}$ is a marked natural number, therefore, $\left\|\left.E\right|_{\operatorname{span}_{\mathrm{K}}\left\{e_{k, l}: k+l>N\right\}}\right\|<\epsilon$. Hence for each $\epsilon>0$ there exists a compact operator $T$ such that from $|\tilde{z} T z|<1$ it follows, $|\widehat{\mu}(y)-\hat{\mu}(x)|<\epsilon$ for each $x-y=z$, where $x, y, z \in Y^{*}$. Therefore, by [24, Theorem 2.30] the characteristic functional $\hat{\mu}$ defines a Radon probability measure on $\mathrm{Bco}(X)$.

Vice versa suppose that each finite-dimensional over $\mathbf{K}$ projection of $\mu$ is a measure of the same type. If for a given one-dimensional over $\mathbf{K}$ subspace $W$ in $X$ there is the equality $B\left(v_{q}(z), v_{q}(z)\right)=0$ for each $z \in W$, then the projection $\mu_{W}$ of $\mu$ is the atomic measure with one atom. Show $B \in L_{1}\left(c_{0}\left(\omega_{0}, \mathbf{K}\right)\right)$ and $\gamma \in c_{0}\left(\omega_{0}, \mathbf{K}\right)$. Let $0 \neq x \in X$ and consider the projection $\pi_{x}: X \rightarrow x \mathbf{K}$. Since $\mu_{x \mathbf{K}}$ is the measure on $\mathrm{Bco}(x \mathbf{K})$, then its characteristic functional satisfies $[24,25,26$, conditions of Theorem 2.30]. Then $\hat{\mu}$ for $x \mathbf{K}$ gives the same characteristic functional of the type

$$
\hat{\mu}_{x \mathbf{K}}(z)=s^{\left[b_{x}\left(v_{q}^{s}(z)\right)^{2}\right]} \chi_{\delta_{x}}(z)
$$

for each $z \in x \mathbf{K}$, where $b_{x}>0$ and $\delta_{x} \in \mathbf{K}$ are constants depending on the parameter $0 \neq x \in X$. Since $x$ and $z$ are arbitrary, then this implies that $B \in L_{1}$ and $\gamma \in c_{0}\left(\omega_{0}, \mathbf{K}\right)$.

Corollary 3.2. A q-Gaussian measure $\mu$ from Proposition 3.1 with $\operatorname{Tr}(B)<\infty$ is quasiinvariant and pseudodifferentiable for some $b \in \mathbb{C}_{\text {s }}$ relative to a dense subspace $J_{\mu} \subset M_{\mu}=$ $\left\{x \in X: v_{q}^{s}(x) \in E^{1 / 2}(Y)\right\}$. Moreover, if $B$ is diagonal, then each one-dimensional projection $\mu^{g}$ has the following characteristic functional:

$$
\hat{\mu}^{g}(h)=s^{\left(\sum_{j} \beta_{j}\left|g_{j}\right|^{q}\right)|h|^{q}} \chi_{g(\gamma)}(h),
$$

where $g=\left(g_{j}: j \in \omega_{0}\right) \in c_{0}\left(\omega_{0}, \mathbf{K}\right)^{*}, \beta_{j}>0$ for each $j$.

Proof. Using the projective limit it is possible to reduce consideration to the Banach space $X$. Take a prime number $s$ such that $s \neq p$ and consider a field $\mathbf{K}_{\mathbf{s}}$ such that $\mathbf{K}$ is compatible with $\mathbf{K}_{\mathbf{s}}$, which is possible, since $\mathbf{K}$ is a finite algebraic extension of $\mathbb{Q}_{\mathbf{p}}$ and it is possible to take in particular $\mathbf{K}_{\mathbf{s}}=\mathbb{Q}_{\mathbf{s}}$. Recall that a group $G$ for which $o(G) \subset o\left(\mathbf{T}_{\mathbf{K}}\right)$ is called compatible with $\mathbf{K}$, where $o(G)$ denotes the set of all natural numbers for which $G$ has an open subgroup $U$ such that at least one of the elements of the quotient group $G / U$ has order $n$, $\mathrm{T}$ denotes the group of all roots of 1 , and $\mathbf{T}_{\mathbf{K}}$ denotes its subgroup of all elements whose 
orders are not divisible by the characteristic $p$ of the residue class field $k$ of $\mathbf{K}$. A character of $G$ is a continuous homomorphism $f: G \rightarrow$ T. Under pointwise multiplication characters form a group denoted by $\hat{G .}$. A group $G$ is called torsional, if each compact subset $V$ of $G$ is contained in a compact subgroup of $G$. In view of [30, Theorem 9.14] $\hat{K^{*}}$ is isomorphic with $\mathbf{K}$. A K-valued character of a group $G$ is a continuous homomorphism $f: G \rightarrow \mathbf{T}_{\mathbf{K}}$. The family of all $\mathbf{K}$-valued characters form a group denoted by $\hat{G_{\mathbf{K}}}$. Since $\mathbf{K}$ is compatible with $\mathbf{K}_{\mathbf{s}}$ and $\lim _{n \rightarrow \infty} p^{n}=0$, then $\hat{\mathbf{K}^{-}}$is isomorphic with $\hat{\mathbf{K}_{\mathbf{K}_{\mathbf{s}}}}$. If $G$ is a torsional group, then the Fourier-Stieltjes transform of a tight measure $\mu \in M(G)$ is the mapping $\hat{\mu}: \hat{G_{\mathrm{K}}} \rightarrow \mathbf{K}$ defined by the formula: $\hat{\mu}(g):=\int_{G} \chi(x) \mu(d x)$, where $\chi \in \hat{G_{\mathrm{K}}}$. In view of [30, Schikhof Theorem 9.21] the Fourier-Stieltjes transformation induces a Banach algebra isomorphism $L(G, \mathscr{R}, w, \mathbf{K})$ with $C_{\infty}\left(\hat{G_{\mathbf{K}}}, \mathbf{K}\right)$, where $w$ is a nontrivial Haar $\mathbf{K}$-valued measure on $G$. Therefore, in this situation there exists the Banach algebra isomorphism of $L\left(\mathbf{K}, \mathscr{R}, w, \mathbf{K}_{\mathbf{s}}\right)$ with $C_{\infty}\left(\hat{\mathbf{K}_{\mathbf{K}_{\mathbf{s}}}}, \mathbf{K}_{\mathbf{s}}\right)$.

Therefore, from the proof above and [24, Theorem 2.30] it follows that the measure $\mu_{q, B, \gamma}$ is quasi-invariant relative to shifts on vectors from the dense subspace $X^{\prime}$ in $X$ such that $X^{\prime}=\left\{x \in X: v_{q}^{s}(x) \in E^{1 / 2}(Y)\right\}$, which is $\mathbf{K}$-linear, since $B$ is $\mathbb{R}$-bilinear and $B(y, z)=$ : $(E y, z)$ for each $y, z \in Y$ and $v_{q}^{s}(a x)=|a|^{q / 2} v_{q}^{s}(x)$ and $v_{q}^{s}\left(x_{j}+t_{j}\right) \leq \max \left(v_{q}^{s}\left(x_{j}\right), v_{q}^{s}\left(t_{j}\right)\right)$ for each $x, t \in X$ and each $a \in \mathbf{K}$, where $E$ is the nondegenerate positive definite of trace class $\mathbb{R}$-linear operator on $Y, x=\sum_{j} x_{j} e_{j}, x_{j} \in \mathbf{K}$, since $l_{2}^{*}=l_{2}$ and $E$ can be extended from $\mathrm{D}_{B, Y}$ on $Y$.

Consider $s^{a+i b}$ as in Section 3. Note, that $\left|\left(|z|_{p}\right)\right|_{s}=1$ for each $z \in \mathbf{K}$, where the field $\mathbf{K}$ is compatible with $\mathbf{K}_{\mathbf{s}}$.

The pseudodifferential operator has the form

$$
\operatorname{PD}(b, f(x)):=\int_{\mathbf{K}}[f(x)-f(y)] s^{(-1-b) \times \operatorname{ord}_{p}(x-y)} w(d y),
$$

where $w$ is the Haar $\mathbf{K}_{\mathbf{s}}$-valued measure on $\operatorname{Bco}(\mathbf{K}), b \in \mathbb{C}_{\mathbf{s}}$, particularly; also for $f(x):=$ $\mu(-x z+A)$ for a given $z \in X^{\prime}, A \in \mathrm{B} \operatorname{co}(X)$, where $x, y \in \mathbf{K}$. Using the Fourier-Stieltjes transform write it in the form: $\operatorname{PD}(b, f(x))=F_{v}^{-1}(\xi(v) \psi(v))$, where $\xi(v):=\left[F_{y}(f(x)-\right.$ $f(y))](v), \psi(v):=\left[F_{y}\left(s^{(-1-b) \times \operatorname{ord}_{p}(y)}\right)\right](v), F_{y}$ means the Fourier-Stieltjes operator by the variable $y$. Denoting $A-x z=: S$ we can consider $f(x)=0$ and $f(y)=\mu((x-y) z+S)$ $-\mu(S)$, since $S \in \operatorname{Bco}(X)$. Then $f(y)=\int_{S}(\mu((x-y)+d g)-\mu(d g))=\int_{S}\left[\rho_{\mu}(y-x, g)-\right.$ $1] \mu(d g)$. The constant function $h(g)=1$ is evidently pseudodifferentiable of order $b$ for each $b \in \mathbb{C}_{s}$. Hence the pseudo-differentiability of $\mu$ of order $b$ follows from the existence of pseudodifferential of the quasi-invariance factor $\rho_{\mu}(y, g+x)$ of order $b$ for $\mu$-almost every $g \in X$. In view of [24, Theorem 3.5] and the Fourier-Stieltjes operator isomorphism of Banach algebras $L\left(\mathbf{K}, \mathscr{R}, w, \mathbf{K}_{\mathbf{s}}\right)$ and $C_{\infty}\left(\hat{\mathbf{K}_{\mathbf{K}_{s}}}, \mathbf{K}_{\mathbf{s}}\right)$ the pseudo-differentiability of $\rho_{\mu}$ follows from the existence of $F^{-1}(\hat{\mu} \psi)$, where $\hat{\mu}$ is the characteristic functional of $\mu$. We have

$$
F(f)(y)=\int_{\mathbf{K}} \chi(x y) f(x) w(d x)=\int_{\mathbf{K}} \chi(z) f(z / y)\left[|y|_{p}\right]^{-1} w(d z)
$$


for each $y \neq 0$, where $x, y, z \in \mathbf{K}$, particularly, for $f(x)=s^{-(1+b) \times \operatorname{ord}_{p}(x)}$ we have $f(z / y)$ $=f(z) f(-y)$ and $F(f)(y)=\Gamma^{\mathrm{K}, s}(1+b) f(-y)|y|_{p}^{-1}$, where

$$
\Gamma^{\mathrm{K}, s}(b):=\int_{\mathbf{K}} \chi(z) s^{-b \times \operatorname{ord}_{p}(x)} w(d z),
$$

$f(-y)=s^{(1+b) \times \operatorname{ord}_{p}(y)}$, since $\operatorname{ord}_{p}(z / y)=\operatorname{ord}_{p}(x)-\operatorname{ord}_{p}(y)$. For a nontrivial character of an order $m \in \mathbb{Z}$ from the definition it follows that $\Gamma^{\mathrm{K}, s}(b) \neq 0$ for each $b$ with $\operatorname{Re}(b) \neq 0$, since $\left|s^{-b n}\right|_{s}=s^{\operatorname{Re}(b) n}$ for each $n \in \mathbb{Z}$. Therefore, $\psi(y)=s^{(1+b) \times \operatorname{ord}_{p}(y)}|y|_{p}^{-1}$, consequently, $|\psi(y)|_{s}=s^{-(1+\operatorname{Re}(b)) \times \operatorname{ord}_{p}(y)}$ for each $y \neq 0$, since $\left|\left(|y|_{p}\right)\right|_{s}=1$. On the other hand, $|\widehat{\mu}(z)|=s^{-B\left(v_{q}^{s}(z), v_{q}^{s}(z)\right)}$ and $F^{-1}(\hat{\mu} \psi)$ exists for each $b \in \mathbb{C}_{\mathrm{s}}$ with $\operatorname{Re}(b)>-1$, since $\operatorname{Tr}(B)<$ $\infty$, which is correct, since $\mathbb{C}_{s}$ is algebraically isomorphic with $\mathbb{C}$ and $\Gamma_{\mathbf{U}_{s}} \supset(0, \infty)$.

Corollary 3.3. Let $X$ be a complete locally $\mathbf{K}$-convex space of separable type over a local field $\mathbf{K}$, then for each constant $q>0$ there exists a nondegenerate symmetric positive definite operator $B \in L_{1}$ such that a q-Gaussian quasi-measure is a measure on $\mathrm{Bco}(X)$ and each one dimensional over $\mathbf{K}$ projection of this measure is absolutely continuous relative to the nonnegative Haar measure on $\mathbf{K}$.

Proof. A space $Y$ from Section 3.1 corresponding to $X$ is a separable locally $\mathbb{R}$-convex space. Therefore, $Y$ in a weak topology is isomorphic with $\mathbb{R}^{N_{0}}$ from which the existence of $B$ follows. For each $\mathbf{K}$-linear finite-dimensional over $\mathbf{K}$ subspace $S$ a projection $\mu^{S}$ of $\mu$ on $S \subset X$ exists and its density $\mu^{S}(d x) / w(d x)$ relative to the nondegenerate $\mathbf{K}_{\mathbf{s}}$-valued Haar measure $w$ on $S$ is the inverse Fourier-Stieltjes transform $F^{-1}\left(\left.\hat{\mu}\right|_{S^{*}}\right)$ of the restriction of $\hat{\mu}$ on $S^{*}$. For $B \in L_{1}$ each one dimensional projection of $\mu$ corresponding to $\hat{\mu}$ has a density that is a continuous function belonging to $L\left(\mathbf{K}, \operatorname{Bco}(\mathbf{K}), w, \mathbf{K}_{\mathbf{s}}\right)$.

Proposition 3.4. Let $\mu_{q, B, \gamma}$ and $\mu_{q, E, \delta}$ be two q-Gaussian measures with correlation operators $B$ and $E$ of class $L_{1}$, then there exists a convolution of these measures $\mu_{q, B, \gamma} * \mu_{q, E, \delta}$, which is a q-Gaussian measure $\mu_{q, B+E, \gamma+\delta}$.

Proof. Since $B$ and $E$ are nonnegative, then $(B+E)(y, y)=B(y, y)+E(y, y) \geq 0$ for each $y \in Y$, that is, $B+E$ is nonnegative. Evidently, $B+E$ is symmetric and of class $L_{1}$. Moreover, $\mu_{q, B+E, \gamma+\delta}$ is defined on the covering ring $\mathrm{U}_{B+E}$ containing the union of covering rings $\mathrm{U}_{B}$ and $\mathrm{U}_{E}$ on which $\mu_{q, B, \gamma}$ and $\mu_{q, E, \delta}$ are defined correspondingly, since $\operatorname{ker}(B+E) \subset$ $\operatorname{ker}(B) \cap \operatorname{ker}(E)$. Therefore, $\mu_{q, B+E, \gamma+\delta}$ is the tight $q$-Gaussian measure together with $\mu_{q, B, \gamma}$ and $\mu_{q, E, \delta}$ in accordance with Proposition 3.1 on the covering ring $\mathscr{R}_{\mu_{q, B+E, \gamma+\delta}}$ which is the completion of the minimal ring generated by $\mathrm{U}_{B+E}$. Since $\hat{\mu}_{q, B+E, \gamma+\delta}=\widehat{\mu}_{q, B, \gamma} \widehat{\mu}_{q, E, \delta}$, then $\mu_{q, B+E, \gamma+\delta}=\mu_{q, B, \gamma} * \mu_{q, E, \delta}$.

3.2. Remark and definition. A measurable space $(\Omega, F)$ with a probability $\mathbf{K}_{\mathbf{s}}$-valued measure $\lambda$ on a covering ring $\mathrm{F}$ of a set $\Omega$ is called a probability space and it is denoted by $(\Omega, F, \lambda)$. Points $\omega \in \Omega$ are called elementary events and values $\lambda(S)$ probabilities of events $S \in \mathrm{F}$. A measurable map $\xi:(\Omega, \mathrm{F}) \rightarrow(X, \mathrm{~B})$ is called a random variable with values in $X$, where $\mathrm{B}$ is a covering ring such that $\mathrm{B} \subset \mathrm{Bco}(X), \mathrm{Bco}(X)$ is the ring of all clopen subsets of a locally $\mathbf{K}$-convex space $X, \xi^{-1}(\mathrm{~B}) \subset \mathrm{F}$, where $\mathbf{K}$ is a non-Archimedean field complete as an ultrametric space. 
The random variable $\xi$ induces a normalized measure $\nu_{\xi}(A):=\lambda\left(\xi^{-1}(A)\right)$ in $X$ and a new probability space $\left(X, \mathrm{~B}, \nu_{\xi}\right)$.

Let $T$ be a set with a covering ring $\mathscr{R}$ and a measure $\eta: \mathscr{R} \rightarrow \mathbf{K}_{\mathbf{s}}$. Consider the following Banach space $L^{q}(T, \mathscr{R}, \eta, H)$ as the completion of the set of all $\mathscr{R}$-step functions $f: T \rightarrow H$ relative to the following norm:

(1) $\|f\|_{\eta, q}:=\sup _{t \in T}\|f(t)\|_{H} N_{\eta}(t)^{1 / q}$ for $1 \leq q<\infty$;

(2) $\|f\|_{\eta, \infty}:=\sup _{1 \leq q<\infty}\|f(t)\|_{\eta, q}$, where $H$ is a Banach space over $\mathbf{K}$.

For $0<q<1$ this is the metric space with the metric

(3) $\rho_{q}(f, g):=\sup _{t \in T}\|f(t)-g(t)\|_{H} N_{\eta}(t)^{1 / q}$.

If $H$ is a complete locally $\mathbf{K}$-convex space, then $H$ is a projective limit of Banach spaces $H=\lim \left\{H_{\alpha}, \pi_{\beta}^{\alpha}, \Upsilon\right\}$, where $\Upsilon$ is a directed set, $\pi_{\beta}^{\alpha}: H_{\alpha} \rightarrow H_{\beta}$ is a K-linear continuous mapping for each $\alpha \geq \beta$, and $\pi_{\alpha}: H \rightarrow H_{\alpha}$ is a $\mathbf{K}$-linear continuous mapping such that $\pi_{\beta}^{\alpha} \circ \pi_{\alpha}=\pi_{\beta}$ for each $\alpha \geq \beta$ (see [28, Section 6.205]). Each norm $p_{\alpha}$ on $H_{\alpha}$ induces a prednorm $\tilde{p}_{\alpha}$ on $H$. If $f: T \rightarrow H$, then $\pi_{\alpha} \circ f=: f_{\alpha}: T \rightarrow H_{\alpha}$. In this case $L^{q}(T, \mathscr{R}, \eta, H)$ is defined as a completion of a family of all step functions $f: T \rightarrow H$ relative to the family of prednorms

$\left(1^{\prime}\right)\|f\|_{\eta, q, \alpha}:=\sup _{t \in T} \tilde{p}_{\alpha}(f(t)) N_{\eta}(t)^{1 / q}, \alpha \in \Upsilon$, for $1 \leq q<\infty$;

$\left(2^{\prime}\right)\|f\|_{\eta, \infty, \alpha}:=\sup _{1 \leq q<\infty}\|f(t)\|_{\eta, q, \alpha}, \alpha \in \Upsilon$, or pseudometrics;

$\left(3^{\prime}\right) \rho_{q, \alpha}(f, g):=\sup _{t \in T} \tilde{p}_{\alpha}(f(t)-g(t)) N_{\eta}(t)^{1 / q}, \alpha \in \Upsilon$, for $0<q<1$.

Therefore, $L^{q}(T, \mathscr{R}, \eta, H)$ is isomorphic with the projective $\operatorname{limit} \lim \left\{L^{q}\left(T, \mathscr{R}, \eta, H_{\alpha}\right)\right.$, $\left.\pi_{\beta}^{\alpha}, \Upsilon\right\}$. For $q=1$ we write simply $L(T, \mathscr{R}, \eta, H)$ and $\|f\|_{\eta}$. This definition is correct, since $\lim _{q \rightarrow \infty} a^{1 / q}=1$ for each $\infty>a>0$. For example, $T$ may be a subset of $\mathbb{R}$. Let $\mathbb{R}_{\mathbf{d}}$ be the field $\mathbb{R}$ supplied with the discrete topology. Since the cardinality $\operatorname{card}(\mathbb{R})=c=2^{\mathrm{N}_{0}}$, then there are bijective mappings of $\mathbb{R}$ on $Y_{1}:=\{0, \ldots, b\}^{\mathbb{N}}$ and also on $Y_{2}:=\mathbb{N}^{\mathbb{N}}$, where $b$ is a positive integer number. Supply the sets $\{0, \ldots, b\}$ and $\mathbb{N}$ with the discrete topologies and $Y_{1}$ and $Y_{2}$ with the product topologies. Then zero-dimensional spaces $Y_{1}$ and $Y_{2}$ supply $\mathbb{R}$ with covering separating rings $\mathscr{R}_{1}$ and $\mathscr{R}_{2}$ contained in $\mathrm{Bco}\left(Y_{1}\right)$ and $\mathrm{Bco}\left(Y_{2}\right)$, respectively. Certainly this is not related with the standard (Euclidean) metric in $\mathbb{R}$. Therefore, for the space $L^{q}(T, \mathscr{R}, \eta, H)$ we can consider $t \in T$ as the real-time parameter. If $T \subset \mathbf{F}$ with a non-Archimedean field $\mathbf{F}$, then we can consider the non-Archimedean time parameter.

If $T$ is a zero-dimensional $T_{1}$-space, then denote by $C_{b}^{0}(T, H)$ the Banach space of all continuous bounded functions $f: T \rightarrow H$ supplied with the norm

(4) $\|f\|_{C^{0}}:=\sup _{t \in T}\|f(t)\|_{H}<\infty$.

If $T$ is compact, then $C_{b}^{0}(T, H)$ is isomorphic with the space $C^{0}(T, H)$ of all continuous functions $f: T \rightarrow H$.

For a set $T$ and a complete locally $\mathbf{K}$-convex space $H$ over $\mathbf{K}$ consider the product K-convex space $H^{T}:=\prod_{t \in T} H_{t}$ in the product topology, where $H_{t}:=H$ for each $t \in T$.

Then take on either $X:=X(T, H)=L^{q}(T, \mathscr{R}, \eta, H)$ or $X:=X(T, H)=C_{b}^{0}(T, H)$ or on $X=X(T, H)=H^{T}$ a covering ring $\mathrm{B}$ such that $\mathrm{B} \subset \mathrm{B} \operatorname{co}(X)$. Consider a random variable $\xi: \omega \mapsto \xi(t, \omega)$ with values in $(X, \mathrm{~B})$, where $t \in T$.

Events $S_{1}, \ldots, S_{n}$ are called independent in totality if $P\left(\prod_{k=1}^{n} S_{k}\right)=\prod_{k=1}^{n} P\left(S_{k}\right)$. Subrings $\mathrm{F}_{k} \subset \mathrm{F}$ are said to be independent if all collections of events $S_{k} \in \mathrm{F}_{k}$ are independent in totality, where $k=1, \ldots, n, n \in \mathbb{N}$. To each collection of random variables $\xi_{\gamma}$ on $(\Omega, \mathrm{F})$ 
with $\gamma \in \Upsilon$ is related the minimal ring $\mathrm{F}_{\Upsilon} \subset \mathrm{F}$ with respect to which all $\xi_{\gamma}$ are measurable, where $\Upsilon$ is a set. Collections $\left\{\xi_{\gamma}: \gamma \in \Upsilon_{j}\right\}$ are called independent if so are $\mathrm{F}_{\Upsilon_{j}}$, where $\Upsilon_{j} \subset \Upsilon$ for each $j=1, \ldots, n, n \in \mathbb{N}$.

Consider $T$ such that card $(T)>n$. For $X=C_{b}^{0}(T, H)$ or $X=H^{T}$ define $X\left(T, H ;\left(t_{1}, \ldots\right.\right.$, $\left.\left.t_{n}\right) ;\left(z_{1}, \ldots, z_{n}\right)\right)$ as a closed submanifold in $X$ of all $f: T \rightarrow H, f \in X$ such that $f\left(t_{1}\right)=$ $z_{1}, \ldots, f\left(t_{n}\right)=z_{n}$, where $t_{1}, \ldots, t_{n}$ are pairwise distinct points in $T$ and $z_{1}, \ldots, z_{n}$ are points in $H$. For $X=L^{q}(T, \mathscr{R}, \eta, H)$ and pairwise distinct points $t_{1}, \ldots, t_{n}$ in $T$ with $N_{\eta}\left(t_{1}\right)>$ $0, \ldots, N_{\eta}\left(t_{n}\right)>0$ define $X\left(T, H ;\left(t_{1}, \ldots, t_{n}\right) ;\left(z_{1}, \ldots, z_{n}\right)\right)$ as a closed submanifold which is the completion relative to the norm $\|f\|_{\eta, q}$ of a family of $\mathscr{R}$-step functions $f: T \rightarrow H$ such that $f\left(t_{1}\right)=z_{1}, \ldots, f\left(t_{n}\right)=z_{n}$. In these cases $X\left(T, H ;\left(t_{1}, \ldots, t_{n}\right) ;(0, \ldots, 0)\right)$ is the proper $\mathbf{K}$ linear subspace of $X(T, H)$ such that $X(T, H)$ is isomorphic with $X\left(T, H ;\left(t_{1}, \ldots, t_{n}\right) ;(0, \ldots\right.$, $0)) \oplus H^{n}$, since if $f \in X$, then $f(t)-f\left(t_{1}\right)=: g(t) \in X\left(T, H ; t_{1} ; 0\right)$ (in the third case we use that $T \in \mathscr{R}$ and hence there exists the embedding $H \hookrightarrow X)$. For $n=1, t_{0} \in T$, and $z_{1}=0$ we denote $X_{0}:=X_{0}(T, H):=X\left(T, H ; t_{0} ; 0\right)$.

3.3. Definitions. We define a (non-Archimedean) stochastic process $w(t, \omega)$ with values in $H$ as a random variable such that

(i) the differences $w\left(t_{4}, \omega\right)-w\left(t_{3}, \omega\right)$ and $w\left(t_{2}, \omega\right)-w\left(t_{1}, \omega\right)$ are independent for each chosen $\left(t_{1}, t_{2}\right)$ and $\left(t_{3}, t_{4}\right)$ with $t_{1} \neq t_{2}, t_{3} \neq t_{4}$, such that either $t_{1}$ or $t_{2}$ is not in the two-element set $\left\{t_{3}, t_{4}\right\}$, where $\omega \in \Omega$;

(ii) the random variable $\omega(t, \omega)-\omega(u, \omega)$ has a distribution $\mu^{F_{t, u}}$, where $\mu$ is a probability $\mathbf{K}_{\mathbf{s}}$-valued measure on $(X(T, H), \mathrm{B})$ from Section $3.2, \mu^{g}(A):=\mu\left(g^{-1}(A)\right)$ for $g: X \rightarrow H$ such that $g^{-1}\left(\mathscr{R}_{H}\right) \subset \mathrm{B}$ and each $A \in \mathscr{R}_{H}$, a continuous linear operator $F_{t, u}: X \rightarrow H$ is given by the formula $F_{t, u}(w):=w(t, \omega)-w(u, \omega)$ for each $w \in L^{q}(\Omega, \mathrm{F}, \lambda ; X)$, where $1 \leq q \leq \infty, \mathscr{R}_{H}$ is a covering ring of $H$ such that $F_{t, u}^{-1}\left(\mathscr{R}_{H}\right) \subset$ B for each $t \neq u$ in $T$;

(iii) we also put $w(0, \omega)=0$, that is, we consider a $\mathrm{K}$-linear subspace $L^{q}\left(\Omega, \mathrm{F}, \lambda ; X_{0}\right)$ of $L^{q}(\Omega, F, \lambda ; X)$, where $\Omega \neq \varnothing, X_{0}$ is the closed subspace of $X$ as in Section 3.2.

Definition 3.5. Let $B$ and $q$ be as in Section 3.1 and denote by $\mu_{q, B, \gamma}$ the corresponding $q$ Gaussian $\mathbf{K}_{\mathrm{s}}$-valued measure on $H$. Let $\xi$ be a stochastic process with a real time $t \in T \subset \mathbb{R}$ (see Section 3.3), then it is called a non-Archimedean $q$-Wiener process with real time (and controlled by $\mathbf{K}_{\mathbf{s}}$-valued measure), if

(ii) ${ }^{\prime}$ the random variable $\xi(t, \omega)-\xi(u, \omega)$ has a distribution $\mu_{q,(t-u) B, \gamma}$ for each $t \neq u \in T$.

Let $\xi$ be a stochastic process with a non-Archimedean time $t \in T \subset \mathbf{F}$, where $\mathbf{F}$ is a local field, then $\xi$ is called a non-Archimedean $q$-Wiener process with F-time (and controlled by $\mathbf{K}_{\mathbf{s}}$-valued measure), if

$(\text { ii) })^{\prime \prime}$ the random variable $\xi(t, \omega)-\xi(u, \omega)$ has a distribution $\mu_{q, \ln \left[\chi_{\mathrm{F}}(t-u)\right] B, \gamma}$ for each $t \neq$ $u \in T$, where $\chi_{\mathbf{F}}: \mathbf{F} \rightarrow \mathbf{T}$ is a continuous character of $\mathbf{F}$ as the additive group (see $[24,25,26$, Section 2.5]).

Proposition 3.6. For each given q-Gaussian measure a non-Archimedean q-Wiener process with real (F resp.) time exists.

Proof. In view of Proposition 3.4, for each $t>u>b$ a random variable $\xi(t, \omega)-\xi(b, \omega)$ has a distribution $\mu_{q,(t-b) B, \gamma}$ for real-time parameter. If $t, u$, and $b$ are pairwise different points 
in $\mathbf{F}$, then $\xi(t, \omega)-\xi(b, \omega)$ has a distribution $\mu_{q, \ln \left[\chi_{\mathbf{F}}(t-b)\right] B, \gamma}$, since $\ln \left[\chi_{\mathbf{F}}(t-u)\right]+\ln \left[\chi_{\mathbf{F}}(u-\right.$ $b)]=\ln \left[\chi_{\mathbf{F}}(t-b)\right]$. This induces the Markov quasimeasure $\mu_{x_{0}, \tau}^{(q)}$ on $\left(\prod_{t \in T}\left(H_{t}, \mathrm{U}_{t}\right)\right)$, where $H_{t}=H$ and $\mathrm{U}_{t}=\mathrm{Bco}(H)$ for each $t \in T$. In view of [24, 25, 26, Theorem 2.39] there exists an abstract probability space $(\Omega, \mathrm{F}, \lambda)$, consequently, the corresponding space $L(\Omega, \mathrm{F}$, $\lambda, \mathbf{K}_{\mathbf{s}}$ ) exists.

Proposition 3.7. Let $\xi$ be a $q$-Gaussian process with values in a Banach space $H=c_{0}(\alpha, \mathbf{K})$, a time parameter $t \in T$ (controlled by a $\mathbf{K}_{\mathbf{s}}$-valued measure), and a positive definite correlation operator $B$ of trace class and $\gamma=0$, where $\operatorname{card}(\alpha) \leq \aleph_{0}$, either $T \subset \mathbb{R}$ or $T \subset \mathbf{F}$. Then either

$$
\lim _{N \in \alpha} M_{t}\left[v_{q}^{s}\left(e^{1}(\xi(t, \omega))\right)\right]^{2}+\cdots+\left[v_{q}^{s}\left(e^{N}(\xi(t, \omega))\right)\right]^{2}=t \operatorname{Tr}(B)
$$

or

$$
\lim _{N \in \alpha} M_{t}\left[v_{q}^{s}\left(e^{1}(\xi(t, \omega))\right)\right]^{2}+\cdots+\left[v_{q}^{s}\left(e^{N}(\xi(t, \omega))\right)\right]^{2}=\left[\ln \left(\chi_{\mathbf{F}}(t)\right)\right] \operatorname{Tr}(B)
$$

respectively.

Proof. Define $\mathbf{U}_{\mathrm{s}}$-valued moments

$$
m_{k}^{q}\left(e^{j_{1}}, \ldots, e^{j_{k}}\right):=\int_{H} v_{2 q}^{s}\left(e^{j_{1}}(x)\right) \cdots v_{2 q}^{s}\left(e^{j_{k}}(x)\right) \mu_{q, B, \gamma}(d x)
$$

for linear continuous functionals $e^{j_{1}}, \ldots, e^{j_{k}}$ on $H$ such that $e^{l}\left(e_{j}\right)=\delta_{j}^{l}$, where $\left\{e_{j}: j \in \alpha\right\}$ is the standard orthonormal base in $H$.

Consider the operator

$$
{ }_{P} \partial^{u} \psi(x):=F^{-1}\left(\hat{f}_{u-1}(y) \hat{\psi}(y)|y|_{p}\right)(x)
$$

where $f_{u}(x):=s^{-(1+u) \times \operatorname{ord}_{p}(x)} / \Gamma^{\mathrm{K}, s}(1+u)$ and $F\left(f_{u}\right)(y)=\Gamma^{\mathrm{K}, s}(1+u) f_{u}(-y)|y|_{p}^{-1}$ (see Corollary 3.2), where $F$ denotes the Fourier-Stieltjes operator defined with the help of the $\mathbf{K}_{\mathbf{s}}$-valued Haar measure $w$ on $\operatorname{Bco}(\mathbf{K}), F(\psi)=: \hat{\psi}, \operatorname{Re}(u) \neq-1$, and $\psi: \mathbf{K} \rightarrow \mathbf{K}_{\mathbf{s}}$. Then

$$
{ }_{P} \partial^{u} f_{b}(x)=F^{-1}\left(\Gamma^{\mathrm{K}, s}(u) f_{u-1}(-y) \Gamma^{\mathrm{K}, s}(1+b) f_{b}(-y)|y|_{p}^{-1}\right)=f_{(u+b)}(x)
$$

for each $u$ with $\operatorname{Re}(u) \neq 0$, since

$$
F^{-1}\left(s^{-(1+u+b) \times \operatorname{ord}_{p}(-y)}|y|_{p}^{-1}\right)(x)=\left(\Gamma^{\mathrm{K}, s}(1+u+b)\right)^{-1} s^{-(1+u+b) \times \operatorname{ord}_{p}(-y)}(x) .
$$

For $u=1$ we write shortly ${ }_{P} \partial^{1}={ }_{P} \partial$ and ${ }_{P} \partial_{j}^{u}$ denotes the operator of partial pseudodifferential (with weight multiplier) given by (3.11) by the variable $x_{j}$. A function $\psi$ for which ${ }_{p} \partial_{j}^{u} \psi$ exists is called pseudodifferentiable (with weight multiplier) of order $u$ by 
variable $x_{j}$. Then

$$
\begin{aligned}
m_{2 k}^{q / 2}\left(e^{j_{1}}, \ldots, e^{j_{2 k}}\right)\left(\Gamma^{\mathrm{K}, s}\left(\frac{q}{2}\right)\right)^{2 k} & :=\int_{H} s^{-q \operatorname{ord}_{p}\left(x_{j_{1}}\right) / 2} \cdots s^{-q \operatorname{ord}_{p}\left(x_{j_{2 k}}\right) / 2} \mu_{q, B, \gamma}(d x) \\
& ={ }_{P} \partial_{j_{1}}^{q / 2} \cdots{ }_{P} \partial_{j_{2 k} / 2}^{q / 2} \widehat{\mu}_{q, B, \gamma}(0) \\
& =\left.\left(\left[\begin{array}{c}
q / 2 \\
\mathrm{PD}
\end{array}\right]^{2 k} \hat{\mu}(x)\right)\right|_{x=0} \cdot\left(e^{j_{1}}, \ldots, e^{j_{2 k}}\right),
\end{aligned}
$$

where $(\stackrel{q}{\mathrm{PD}} f(x)) \cdot e^{j}:={ }_{P} \partial_{j} f(x)$. Therefore,

$$
\begin{aligned}
m_{2 k}^{q / 2}\left(e^{j_{1}}, \ldots, e^{j_{2 k}}\right)\left(\Gamma^{\mathrm{K}, s}\left(\frac{q}{2}\right)\right)^{2 k} & =(k !)^{-1}\left[\begin{array}{l}
q / 2 \\
\mathrm{PD}
\end{array}\right]^{2 k}\left[B\left(v_{q}^{s}(z), v_{q}^{s}(z)\right)\right]^{k} \cdot\left(e_{j_{1}}, \ldots, e_{j_{2 k}}\right) \\
& =(k !)^{-1} \sum_{\sigma \in \Sigma_{2 k}} B_{\sigma\left(j_{1}\right), \sigma\left(j_{2}\right)} \cdots B_{\sigma\left(j_{2 k-1}\right), \sigma\left(j_{2 k}\right)},
\end{aligned}
$$

since $\gamma=0$ and $\chi_{\gamma}(z)=1$, where $\Sigma_{k}$ is the symmetric group of all bijective mappings $\sigma$ of the set $\{1, \ldots, k\}$ onto itself, $B_{l, j}:=B\left(e_{j}, e_{l}\right)$, since $Y^{*}=Y$ for $Y=l_{2}(\alpha, \mathbb{R})$. Therefore, for each $B \in L_{1}$ and $A \in L_{\infty}$ we have

$$
\int_{H} A\left(v_{q}(x), v_{q}(x)\right) \mu_{q, B, 0}(d x)=\lim _{N \in \alpha} \sum_{j=1}^{N} \sum_{k=1}^{N} A_{j, k} m_{2}^{q / 2}\left(e_{j}, e_{k}\right)=\operatorname{Tr}(A B),
$$

since $\mathbb{C}_{\mathbf{s}} \subset \mathbf{U}_{\mathbf{s}}$ and algebraically $\mathbb{C}_{\mathbf{s}}$ is isomorphic with $\mathbb{C}$.

In particular for $A=I$ and $\mu_{q, t B, 0}$ corresponding to the transition measure of $\xi(t, \omega)$ we get Formula (3.8) for a real-time parameter, using $\mu_{q, \ln \left[\chi_{\mathrm{F}}(t)\right] B, 0}$ we get Formula (3.9) for a time parameter belonging to $\mathbf{F}$, since $\xi\left(t_{0}, \omega\right)=0$ for each $\omega$.

Corollary 3.8. Let $H=\mathbf{K}$ and $\xi, B=1$, and $\gamma$ be as in Proposition 3.7, then

$$
M\left[\int_{t \in[a, b]} \phi(t, \omega) v_{2 q}^{s}(d \xi(t, \omega))\right]=M\left[\int_{a}^{b} \phi(t, \omega) d t\right]
$$

for each $a<b \in T$ with real time, where $\phi(t, \omega) \in L\left(\Omega, U, \lambda, C_{0}^{0}(T, \mathbb{R})\right) \xi \in L\left(\Omega, U, \lambda, X_{0}(T\right.$, $\mathbf{K})),(\Omega, \mathrm{U}, \lambda)$ is a probability measure space.

Proof. Since

$$
\int_{t \in[a, b]} \phi(t, \omega) v_{2 q}^{s}(d \xi(t, \omega))=\lim _{\max _{j}\left(t_{j+1}-t_{j}\right) \rightarrow 0} \sum_{j=1}^{N} \phi\left(t_{j}, \omega\right) v_{q}^{s}\left(\xi\left(t_{j+1}, \omega\right)-\xi\left(t_{j}, \omega\right)\right)
$$

for $\lambda$-almost all $\omega \in \Omega$, since $\mathbb{C}_{\mathbf{s}} \subset \mathbf{U}_{\mathbf{s}}$ and $\mathbb{C}_{\mathbf{s}}$ is algebraically isomorphic with $\mathbb{C}$, then from the application of Formula (3.8) to each $v_{2 q}^{s}\left(\xi\left(t_{j+1}, \omega\right)-\xi\left(t_{j}, \omega\right)\right)$ and the existence of the limit by finite partitions $a=t_{1}<t_{2}<\cdots<t_{N+1}=b$ of the segment $[a, b]$ it follows Formula (3.17). 


\section{Definitions and notes}

Consider a pseudodifferential operator on $H=c_{0}(\alpha, \mathbf{K})$ such that

$$
\mathrm{A}=\sum_{0 \leq k \in \mathbb{Z} ; j_{1}, \ldots, j_{k} \in \alpha}(-i)^{k} b_{j_{1}, \ldots, j_{k} P}^{k} \partial_{j_{1}} \cdots{ }_{P} \partial_{j_{k}},
$$

where $b_{j_{1}, \ldots, j_{k}}^{k} \in \mathbb{R},{ }_{P} \partial_{j_{k}}:={ }_{P} \partial_{j_{k}}^{1}$. If there exists $n:=\max \left\{k: b_{j_{1}, \ldots, j_{k}}^{k} \neq 0, j_{1}, \ldots, j_{k} \in \alpha\right\}$, then $n$ is called an order of $A$, Ord $(A)$, where ${ }_{P} \partial_{j}$ is defined by formula (3.11). If $A=0$, then by definition $\operatorname{Ord}(\mathrm{A})=0$. If there is not any such finite $n$, then $\operatorname{Ord}(\mathrm{A})=\infty$. We suppose that the corresponding form $\tilde{A}$ on $\bigoplus_{k} Y^{k}$ is continuous into $\mathbb{C}$, where

$$
\tilde{A}(y)=-\sum_{0 \leq k \in \mathbb{Z} ; j_{1}, \ldots, j_{k} \in \alpha} \frac{(-i)^{k} b_{j_{1}, \ldots, j_{k}}^{k} y_{j_{1}} \cdots y_{j_{k}}}{\ln s}
$$

$y \in l_{2}(\alpha, \mathbb{R})=: Y$. If $\tilde{A}(y)>0$ for each $y \neq 0$ in $Y$, then $\mathrm{A}$ is called strictly elliptic pseudodifferential operator.

Let $X$ be a complete locally $\mathbf{K}$-convex space, and let $Z$ be a complete locally $\mathbf{U}_{\mathrm{s}}$-convex space. For $0 \leq n \in \mathbb{R}$ a space of all functions $f: X \rightarrow Z$ such that $f(x)$ and $\left({ }_{\mathrm{PD}}^{k} f(x)\right)$. $\left(y^{1}, \ldots, y^{l(k)}\right)$ are continuous functions on $X$ for each $y^{1}, \ldots, y^{l(k)} \in\left\{e^{1}, e^{2}, e^{3}, \ldots\right\} \subset X^{*}$, $l(k):=[k]+\operatorname{sign}\{k\}$ for each $k \in \mathbb{N}$ such that $k \leq[n]$ and also for $k=n$ is denoted by ${ }_{P} \mathscr{C}^{n}(X, Z)$, and $f \in{ }_{P} \mathscr{C}^{n}(X, Z)$ is called $n$ times continuously pseudodifferentiable, where $[n] \leq n$ is an integer part of $n, 1>\{n\}:=n-[n] \geq 0$ is a fractional part of $n$. Then ${ }_{P} \mathscr{C}^{\infty}(X, Z):=\bigcap_{n=1}^{\infty} P^{C^{n}}(X, Z)$ denotes a space of all infinitely pseudodifferentiable functions.

Embed $\mathbb{R}$ into $\mathbb{C}_{s}$ and consider the function $v_{2}^{s}: \mathbf{U}_{\mathbf{p}} \rightarrow \mathbb{R} \subset \mathbb{C}_{\mathbf{s}}$, then for $t=v_{2}^{s}(\theta), \theta \in$ $\mathbf{K} \subset \mathbf{U}_{\mathbf{p}}$, put $\partial_{t} u(t, x):=\lim _{\theta, \mathbf{K}, \theta \in \mathbf{K}, v_{2}^{s}(\theta) \rightarrow t} \partial_{\theta} u\left(v_{2}^{s}(\theta), x\right)$ for $t \geq 0$, when it exists by the filter of local subfields $\mathbf{K}$ in $\mathbb{C}_{\mathbf{p}}$, which is correct, since $v_{2}^{s}\left(\mathbf{U}_{\mathbf{p}}\right)=[0, \infty), \bigcup_{\mathbf{K} \subset \mathbb{C}_{\mathbf{p}}} \mathbf{K}$ is dense in $\mathbb{C}_{\mathbf{p}}, \Gamma_{\mathbb{C}_{\mathrm{p}}}=(0, \infty) \cap \mathbb{Q}$.

Theorem 4.1. Let A be a strictly elliptic pseudodifferential operator on $H=c_{0}(\alpha, \mathbf{K})$, $\operatorname{card}(\alpha) \leq \mathrm{N}_{0}$, and let $t \in T=[0, b] \subset \mathbb{R}$. Suppose also that $u_{0}(x-y) \in L\left(H, \operatorname{Bco}(H), \mu_{t \tilde{A}}\right.$, $\left.\mathbf{U}_{s}\right)$ for each marked $y \in H$ as a function by $x \in H, u_{0}(x) \in_{P} \mathscr{C}^{\operatorname{Ord}(\mathrm{A})}\left(H, \mathbf{U}_{s}\right)$. Then the non-Archimedean analog of the Cauchy problem

$$
\partial_{t} u(t, x)=\mathrm{A} u, \quad u(0, x)=u_{0}(x)
$$

has a solution given by

$$
u(t, x)=\int_{H} u_{0}(x-y) \mu_{t \tilde{A}}(d y)
$$

where $\mu_{t \tilde{A}}$ is a $\mathbf{K}_{\mathbf{s}}$-valued measure on $H$ with a characteristic functional $\widehat{\mu}_{t} \tilde{A}(z):=s^{t \tilde{A}\left(v_{2}^{s}(z)\right)}$.

Proof. In accordance with Sections 3 and 4 we have $Y=l_{2}(\alpha, \mathbb{R})$. The function $s^{t \tilde{A}\left(v_{2}^{s}(z)\right)}$ is continuous on $H \hookrightarrow H^{*}$ for each $t \in \mathbb{R}$ such that the family $H$ of continuous K-linear functionals on $H$ separates points in $H$. In view of [24, Theorem 2.30], which defines 
a tight measure on $H$ for each $t>0$. The functional $\tilde{A}$ on each ball of radius $0<R<\infty$

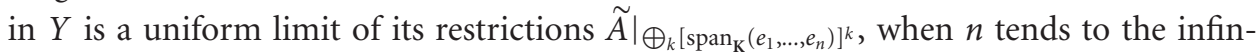
ity, since $\tilde{A}$ is continuous on $\bigoplus_{k} Y^{k}$. Since $u_{0}(x-y) \in L\left(H, \operatorname{Bco}(H), \mu_{t}, \mathbf{U}_{s}\right)$ and a space of cylindrical functions is dense in the latter Banach space over $\mathbf{U}_{s}$, then in view of [30, Theorems 9.14, 9.21] and the Fubini theorem it follows that $\lim _{P \rightarrow I} \mathrm{~F}_{P x} u_{0}(P x) \widehat{\mu}_{t}(y+P x)$ converges in $L\left(H, \operatorname{Bco}(H), \mu_{t \tilde{A}}, \mathbf{U}_{s}\right)$ for each $t$, since $\mu_{t_{1} \tilde{A}} * \mu_{t_{2} \tilde{A}}=\mu_{\left(t_{1}+t_{2}\right) \tilde{A}}$ for each $t_{1}, t_{2}$, and $t_{1}+t_{2} \in T$, where $P$ is a projection on a finite-dimensional over $\mathbf{K}$ subspace $H_{P}:=$ $P(H)$ in $H, H_{P} \hookrightarrow H, P$ tends to the unit operator $I$ in the strong operator topology, and $F_{P x} u_{0}(P x)$ denotes a Fourier transform by the variable $P x \in H_{P}$. Consider a function $v:=$ $F_{x}(u)$, then $\partial_{t} v(t, x)=-\tilde{A}\left(v_{2}^{s}(x)\right) v(t, x) \ln s$, consequently, $v(t, x)=v_{0}(x) s^{t \tilde{A}\left(v_{2}^{s}(x)\right)}$. From $u(t, x)=F_{x}^{-1}(v(t, x))$, where $F_{x}(u(t, x))=\lim _{n \rightarrow \infty} F_{x_{1}, \ldots, x_{n}} u(t, x)$, it follows that $u(t, x)=$ $u_{0}(x) *\left[F_{x}^{-1}\left(\hat{\mu}_{t \tilde{A}}\right)\right]=\int_{H} u_{0}(x-y) \mu_{t \tilde{A}}(d y)$, since $u_{0}(x-y) \in L\left(H, \operatorname{Bco}(H), \mu_{t \tilde{A}}, \mathbf{U}_{s}\right)$ and $\mu_{t} \tilde{A}$ is the tight measure on $\mathrm{Bco}(H)$.

Note 4.2. In the particular case of $\operatorname{Ord}(\mathrm{A})=2$ and $\tilde{A}$ corresponding to the Laplace operator, that is, $\tilde{A}(y)=\sum_{l, j} g_{l, j} y_{l} y_{j},(4.3)$ is (the non-Archimedean analog of) the heat equation on $H$.

For $\operatorname{Ord}(\mathrm{A})<\infty$ the form $\tilde{A}_{0}(y)$ corresponding to sum of terms with $k=\operatorname{Ord}(\mathrm{A})$ in Formula (4.2) is called the principal symbol of the operator A. If $\tilde{A}_{0}(y)>0$ for each $y \neq 0$, then $\mathrm{A}$ is called an elliptic pseudodifferential operator. Evidently, Theorem 4.1 is true for elliptic $\mathrm{A}$ of $\operatorname{Ord}(\mathrm{A})<\infty$.

\section{Remark and definitions}

Let the linear spaces $X$ over $\mathbf{K}$ and $Y$ over $\mathbb{R}$ be as in Corollary 3.2 and $B$ be a symmetric nonnegative definite (bilinear) operator on a dense $\mathbb{R}$-linear subspace $\mathrm{D}_{B, Y}$ in $Y^{*}$. A quasi-measure $\mu$ with a characteristic functional

$$
\widehat{\mu}(\zeta, x):=s^{\zeta B\left(v_{q}^{s}(z), v_{q}^{s}(z)\right)} \chi_{\gamma}(z)
$$

for a parameter $\zeta \in \mathbb{C}_{\mathrm{s}}$ with $\operatorname{Re}(\zeta) \geq 0$ defined on $\mathrm{D}_{q, B, X}$ is called an $\mathbf{U}_{\mathrm{s}}$-valued (nonArchimedean analog of Feynman) quasi-measure and we denote it by $\mu_{q, \zeta B, \gamma}$ also, where $\mathrm{D}_{q, B, X}:=\left\{z \in X^{*}:\right.$ there exists $j \in \Upsilon$ such that $\left.z(x)=z_{j}\left(\phi_{j}(x)\right) \forall x \in X, v_{q}^{s}(z) \in \mathrm{D}_{B, Y}\right\}$.

Proposition 5.1. Let $X=\mathrm{D}_{q, B, X}$ and $B$ be positive definite, then for each function $f(z):=$ $\int_{X} \chi_{z}(x) \nu(d x)$ with an $\mathrm{U}_{\mathrm{s}}$-valued tight measure $v$ of finite norm and each $\operatorname{Re}(\zeta)>0$ there exists

$$
\int_{X} f(z) \mu_{\zeta B}(d z)=\lim _{P \rightarrow I} \int_{X} f(P z) \mu_{\zeta B}^{(P)}(d z)=\int_{X} s^{\left(\zeta B\left(v_{q}(z), v_{q}(z)\right)\right)} \chi_{\gamma}(z) \nu(d z)
$$

where $\mu^{(P)}\left(P^{-1}(A)\right):=\mu\left(P^{-1}(A)\right)$ for each $A \in \mathrm{Bco}\left(X_{P}\right), P: X \rightarrow X_{P}$ is a projection on a $\mathbf{K}$ linear subspace $X_{P}$, a convergence $P \rightarrow I$ is considered relative to a strong operator topology. 


\section{Quasi-invariant descending at infinity measures}

Proof. From the use of the projective limit decomposition of $X$ and $[24,25,26$, Theorem 2.37] it follows that there exists

$$
\int_{X} f(z) \mu_{\zeta B}(d z)=\lim _{P \rightarrow I} \int_{X} f(P z) \mu_{\zeta B}^{(P)}(d z)
$$

Then for each finite-dimensional over $\mathbf{K}$ subspace $X_{P}$

$$
\int_{X} f(P z) \mu_{\zeta B}^{(P)}(d z)=\left.\int_{X_{P}}\left\{s^{\zeta B\left(v_{q}^{s}(z), v_{q}^{s}(z)\right)} \chi_{\gamma}(z)\right\}\right|_{X_{P}} v^{X_{P}}(d z)
$$

since $v$ is tight and hence each $v^{X_{P}}$ is tight. Each measure $v_{j}$ is tight, then due to $[24,25,26$, Lemma 2.3 and Section 2.5] there exists the limit

$$
\left.\lim _{P \rightarrow I} \int_{X_{P}}\left\{s^{\zeta B\left(v_{q}^{s}(z), v_{q}^{s}(z)\right)} \chi_{\gamma}(z)\right\}\right|_{X_{P}} v^{X_{P}}(d z)=\int_{X} s^{\zeta B\left(v_{q}^{s}(z), v_{q}^{s}(z)\right)} \chi_{\gamma}(z) \nu(d z)
$$

Proposition 5.2. If conditions of Proposition 5.1 are satisfied and

$$
f(P x) \in L\left(X_{P}, \operatorname{Bco}\left(w^{X_{P}}\right), \mathbf{U}_{\mathbf{s}}\right)
$$

for each finite-dimensional over $\mathbf{K}$ subspace $X_{P}$ in $X$ and

$$
\lim _{R \rightarrow \infty} \sup _{|x| \leq R}|f(x)|=0
$$

then Formula (5.2) is accomplished for $\zeta$ with $\operatorname{Re}(\zeta)=0$, where $w^{X_{P}}$ is a nondegenerate $\mathbf{K}_{\mathbf{s}}$-valued Haar measure on $X_{P}$.

Proof. In view of $[24,25,26$, Theorem 2.37] for the consistent family of measures $\left\{f(P x) \mu_{q, i B, \gamma}^{X_{P}}(d P x): P\right\}$ (see $[24,25,26$, Section 2.36]) there exists a measure on $(X, \mathscr{R})$, where projection operators $P$ are associated with a chosen basis in $X$. The finite-dimensional over $\mathbf{K}$ distribution $\mu_{q, i B, \gamma}^{X_{P}} / w^{X_{P}}(d x)=\left.F^{-1}\left(\hat{\mu}_{q, i B, \gamma}\right)\right|_{X_{P}}$ is in $C_{\infty}\left(X_{P}, \mathbf{U}_{\mathbf{s}}\right)$ due to [30, Theorem 9.21], since $\hat{\mu} \in L\left(X_{P}, \mathrm{~B} \operatorname{co}\left(X_{p}\right), w^{X_{P}}, \mathbf{U}_{\mathbf{s}}\right)$. In view of conditions (5.6), (5.7) above, and the Fubini theorem and using the Fourier-Stieltjes transform we get Formulas (5.2), (5.3). From taking the limit by $P \rightarrow I$ Formula (5.2) follows. This means that $\mu_{q, \zeta B, \gamma}$ exists in the sense of distributions.

Remark 5.3. Put

$$
F \int_{X} f(x) \mu_{q, i B, \gamma}(d x):=\lim _{\zeta \rightarrow i} \int_{X} f(x) \mu_{q, \zeta B, \gamma}(d x)
$$

if such limit exists. If conditions of Proposition 5.2 are satisfied, then $\psi(\zeta):=$ $\int_{X} f(x) \mu_{q, \zeta B, \gamma}(d x)$ is the pseudodifferentiable of order 1 function by $\zeta$ on the set $\{\zeta \in$ $\left.\mathbb{C}_{s}: \operatorname{Re}(\zeta)>0\right\}$ and it is continuous on the subset $\left\{\zeta \in \mathbb{C}_{s}: \operatorname{Re}(\zeta) \geq 0\right\}$, consequently,

$$
F \int_{X} f(x) \mu_{q, i B, \gamma}(d x)=\int_{X} s^{\left\{i B\left(v_{q}^{s}(x), v_{q}^{s}(x)\right)\right\}} \chi_{\gamma}(x) \nu(d x) .
$$


Above non-Archimedean analogs of Gaussian measures with specific properties were defined. Nevertheless, there do not exist usual Gaussian $\mathbf{K}_{\mathbf{s}}$-valued measures on nonArchimedean Banach spaces.

Theorem 5.4. Let $X$ be a Banach space of separable type over a locally compact nonArchimedean field $\mathbf{K}$. Then on $\mathrm{Bco}(X)$ there does not exist a nontrivial $\mathbf{K}_{\mathbf{s}}$-valued (probability) usual Gaussian measure.

Proof. Let $\mu$ be a nontrivial usual Gaussian $\mathbf{K}_{\mathbf{s}}$-valued measure on $\mathrm{B} \operatorname{co}(X)$. Then by the definition its characteristic functional $\hat{\mu}$ must be satisfying $[24,25,26$, conditions 2.5. $(3,5)] \mathbf{U}_{\text {s }}$-valued function and $\lim _{|y| \rightarrow \infty} \hat{\mu}(y)=0$ for each $y \in X^{*} \backslash\{0\}$, where $X^{*}$ is the topological conjugate space to $X$ of all continuous $\mathbf{K}$-linear functionals $f: X \rightarrow \mathbf{K}$. Moreover, there exist a K-bilinear functional $g$ and a compact nondgenerate K-linear operator $T: X^{*} \rightarrow X^{*}$ with $\operatorname{ker}(T)=\{0\}$ and a marked vector $x_{0} \in X$ such that $\hat{\mu}_{x_{0}}(y)=$ $f(g(T y, T y))$ for each $y \in X^{*}$, where $\mu_{x_{0}}(d x):=\mu\left(-x_{0}+d x\right), x \in X$. Since $\mathbf{K}$ is locally compact, then $X^{*}$ is nontrivial and separates points of $X$ (see $\left.[28,30]\right)$. Each onedimensional over $\mathbf{K}$ projection of a Gaussian measure is a Gaussian measure and products of Gaussian measures are Gaussian measures, hence convolutions of Gaussian measures are also Gaussian measures. Therefore, $\hat{\mu}_{x_{0}}: X^{*} \rightarrow \mathbf{U}_{s}$ is a nontrivial character: $\hat{\mu}_{x_{0}}\left(y_{1}+\right.$ $\left.y_{2}\right)=\hat{\mu}_{x_{0}}\left(y_{1}\right) \hat{\mu}_{x_{0}}\left(y_{2}\right)$ for each $y_{1}$ and $y_{2}$ in $X^{*}$. If $\operatorname{char}(\mathbf{K})=0$ and $\mathbf{K}$ is a non-Archimedean field, then there exists a prime number $p$ such that $\mathbb{Q}_{\mathbf{p}}$ is the subfield of $\mathbf{K}$. Then $\widehat{\mu}\left(p^{n} y\right)=$ $(\hat{\mu}(y))^{p^{n}}$ for each $n \in \mathbb{Z}$ and $y \in X^{*} \backslash\{0\}$, particularly, for $n \in \mathbb{N}$ tending to the infinity we have $\lim _{n \rightarrow \infty} p^{n} y=0$ and $\lim _{n \rightarrow \infty} \hat{\mu}_{x_{0}}\left(p^{n} y\right)=1, \lim _{n \rightarrow \infty}\left(\hat{\mu}_{x_{0}}(y)\right)^{p^{n}}=0$, since $s \neq p$ are primes, $\lim _{n \rightarrow \infty} \hat{\mu}_{x_{0}}\left(p^{-n} y\right)=0$ and $\left|\hat{\mu}_{x_{0}}(y)\right|<1$ for $y \neq 0$. This gives the contradiction, hence $\mathbf{K}$ cannot be a non-Archimedean field of zero characteristic. Suppose that $\mathbf{K}$ is a non-Archimedean field of characteristic $\operatorname{char}(\mathbf{K})=p>0$, then $\mathbf{K}$ is isomorphic with the field of formal power series in variable $t$ over a finite field $\mathbf{F}_{\mathbf{p}}$. Therefore, $\hat{\mu}_{x_{0}}(p y)=1$, but $\hat{\mu}_{x_{0}}(y)^{p} \neq 1$ for $y \neq 0$, since $\lim _{n \rightarrow \infty} \hat{\mu}_{x_{0}}\left(t^{-n} y\right)=0$. This contradicts the fact that $\hat{\mu}_{x_{0}}$ need to be the nontrivial character, consequently, $\mathbf{K}$ cannot be a non-Archimedean field of nonzero characteristic as well. It remains the classical case of $X$ over $\mathbb{R}$ or $\mathbb{C}$, but the latter case reduces to $X$ over $\mathbb{R}$ with the help of the isomorphism of $\mathbb{C}$ as the $\mathbb{R}$-linear space with $\mathbb{R}^{2}$.

Theorem 5.5. Let $\mu_{q, B, \gamma}$ and $\mu_{q, B, \delta}$ be two q-Gaussian $\mathbf{K}_{\mathbf{s}}$-valued measures. Then $\mu_{q, B, \gamma}$ is equivalent to $\mu_{q, B, \delta}$ or $\mu_{q, B, \gamma} \perp \mu_{q, B, \delta}$ according to $v_{q}^{s}(\gamma-\delta) \in B^{1 / 2}\left(\mathrm{D}_{B, Y}\right)$, or not. The measure $\mu_{q, B, \gamma}$ is orthogonal to $\mu_{g, B, \delta}$, when $q \neq g$. Two measures $\mu_{q, B, \gamma}$ and $\mu_{g, A, \delta}$ with positive definite nondegenerate $A$ and $B$ are either equivalent or orthogonal.

Theorem 5.6. The measures $\mu_{q, B, \gamma}$ and $\mu_{q, A, \gamma}$ are equivalent if and only if there exists a positive definite bounded invertible operator $T$ such that $A=B^{1 / 2} T B^{1 / 2}$ and $T-I \in L_{2}\left(Y^{*}\right)$.

Proof. Using the projective limit we can reduce consideration to the Banach space $X$. Let $z \in X$ be a marked vector and $P_{z}$ be a projection operator on $z \mathbf{K}$ such that $P_{z}^{2}=P_{z}$, $z=\sum_{j} z_{j} e_{j}$, then the characteristic functional of the projection $\mu_{q, B, \gamma}^{z \mathrm{~K}}$ of $\mu_{q, B, \gamma}$ has the form $\hat{\mu}_{q, B, \gamma}^{z \mathbf{K}}=s^{\left[\left(\sum_{i, j} B_{i, j} v_{q}^{s}\left(z_{i}\right) v_{q}^{s}\left(z_{j}\right)\right) v_{2 q}^{s}(\xi)\right]} \chi_{\gamma(z)}(\xi)$ for each vector $x=\xi z$, where each $z_{j}$ and $\xi \in \mathbf{K}$, since $v_{2 q}^{s}(\xi)=\left(v_{q}^{s}(\xi)\right)^{2}$. Choose a sequence $\left\{{ }_{n} z: n\right\}$ in $X$ such that it is the orthonormal 
basis in $X$ and the operator $G: X \rightarrow X$ such that $G_{n} z={ }_{n} a_{n} z$ with ${ }_{n} a \neq 0$ for each $n \in \mathbb{N}$ and there exists $G^{-1}: G(X) \rightarrow X$ such that it induces the operator $C$ on a dense subspace $\mathscr{D}(Y)$ in $Y$ such that $C B C: Y \rightarrow Y$ is invertible and $\|C B C\|$ and $\left\|(C B C)^{-1}\right\| \in\left[|\pi|,|\pi|^{-1}\right]$. Then

$$
\frac{\mu_{q, A, \gamma}(d x)}{\mu_{q, B, \gamma}(d x)}=\lim _{n \rightarrow \infty}\left[\frac{\mu_{q, A, \gamma}^{V_{n}}\left(d x^{n}\right)}{\lambda^{V_{n}}\left(d x^{n}\right)}\right]\left[\frac{\mu_{q, B, \gamma}^{V_{n}}\left(d x^{n}\right)}{\lambda^{V_{n}}\left(d x^{n}\right)}\right]^{-1},
$$

where $V_{n}:=\operatorname{span}_{\mathbf{K}}\left({ }_{j} z: j=1, \ldots, n\right), x_{n} \in V_{n}$. Consider $x_{n}=G^{-1}\left(y_{n}\right)$, where $y_{n} \in G\left(V_{n}\right)$, then

$$
\left[\frac{\mu_{q, B, \gamma}^{V_{n}}\left(G^{-1} d y^{n}\right)}{\lambda^{V_{n}}\left(G^{-1} d y^{n}\right)}\right], \quad\left[\frac{\mu_{q, B, \gamma}^{V_{n}}\left(G^{-1} d y^{n}\right)}{\lambda^{V_{n}}\left(G^{-1} d y^{n}\right)}\right]^{-1}
$$

are in $L\left(\lambda^{V_{n}}\left(G^{-1} d y^{n}\right)\right)$ for each $n$ such that there exists $m \in \mathbb{N}$ for which

$$
\left\|\left[\frac{\mu_{q, B, \gamma}^{V_{n}}\left(G^{-1} d y^{n}\right)}{\lambda^{V_{n}}\left(G^{-1} d y^{n}\right)}\right]\right\|,\left\|\left[\frac{\mu_{q, B, \gamma}^{V_{n}}\left(G^{-1} d y^{n}\right)}{\lambda^{V_{n}}\left(G^{-1} d y^{n}\right)}\right]^{-1}\right\| \in\left[|\pi|,|\pi|^{-1}\right]
$$

for each $n>m$, where $\|*\|$ is taken in $L\left(\lambda^{V_{n}}\left(G^{-1} d y^{n}\right)\right)$. Then $N_{\mu_{q, C B C, \gamma G^{-1}}^{V_{n}}}\left(y^{n}\right) \in\left[|\pi|,|\pi|^{-1}\right]$ for each $n>m$. Then the existence of $\mu_{q, A, \gamma}(d x) / \mu_{q, B, \gamma}(d x) \in L\left(\mu_{q, B, \gamma}\right)$ is provided by using operator $G$ and the consideration of characteristic functionals of measures, [24, Theorem 3.5] and the fact that the Fourier-Stieltjes transform $F$ is the isomorphism of Banach algebras $L\left(\mathbf{K}, \operatorname{Bco}(\mathbf{K}), v, \mathbf{U}_{s}\right)$ with $C_{\infty}\left(\mathbf{K}, \mathbf{U}_{s}\right)$, where $v$ denotes the Haar normalized by $v(B(\mathbf{K}, 0,1))=1 \mathbf{K}_{\mathbf{s}}$-valued measure on $\mathbf{K}$. If $g \neq q$ then the measure $\mu_{q, B, \gamma}$ is orthogonal to $\mu_{g, B, \delta}$, since

$$
\lim _{R>0, R+n \rightarrow \infty} \sup _{x \in X_{R, n}^{c}}\left|\frac{\left(\mu_{q, B, \gamma}\right)_{X_{n}}}{\left(\mu_{g, B, \delta}\right)_{X_{n}}}\right|(x)=0
$$

for each $q>g$ due to Formula (3.6), where $X_{n}:=\operatorname{span}_{\mathbf{K}}\left(e_{m}: m=n, n+1, \ldots, 2 n\right), X_{R, n}^{c}:=$ $X_{n} \backslash B\left(X_{n}, 0, R\right),\left(\mu_{q, B, \gamma}\right)_{X_{n}}$ is the projection of the measure $\mu_{q, B, \gamma}$ on $X_{n}$. Each term $\beta_{j}$ in $[24$, Theorem 3.5] is in $[0,1] \subset \mathbb{R}$, consequently, the product in this theorem is either converging to a positive constant or diverging to zero, hence two measures $\mu_{q, B, \gamma}$ and $\mu_{g, A, \delta}$ are either equivalent or orthogonal.

Theorem 5.7. Let $X$ be a Banach space of separable type over a locally compact nonArchimedean field $\mathbf{K}$ and $J$ be a dense proper $\mathbf{K}$-linear subspace in $X$ such that the embedding operator $T: J \hookrightarrow X$ is compact and nondegenerate, $\operatorname{ker}(T)=\{0\}$. Then a set $M(X, J)$ of probability $\mathrm{K}_{\mathrm{s}}$-valued measures $\mu$ on $\mathrm{Bco}(X)$ quasi-invariant relative to $J$ is of cardinality $\operatorname{card}\left(\mathbf{K}_{\mathbf{s}}\right)^{\mathrm{c}}$. If $J^{\prime}, J^{\prime} \subset J$, is also a dense $\mathbf{K}$-linear subspace in $X$, then $\mathcal{M}\left(X, J^{\prime}\right) \supset \mathcal{M}(X, J)$. 
Proof. Since $X$ is of separable type over $\mathbf{K}$, then we can choose for a given compact operator $T$ an orthonormal base in $X$ in which $T$ is diagonal and $X$ is isomorphic with $c_{0}$ over $\mathrm{K}$ such that in its standard base $\left\{e_{j}: j \in \mathbb{N}\right\}$ the operator $T$ has the form $T e_{j}=a_{j} e_{j}$, $0 \neq a_{j} \in \mathbf{K}$ for each $j \in \mathbb{N}, \lim _{j \rightarrow \infty} a_{j}=0$. As in [24, 25, 26, Theorem 3.15] take $g_{n} \in$ $L\left(\mathbf{K}, \mathrm{Bco}(\mathbf{K}), w^{\prime}\left(d x / a_{n}\right), \mathbf{K}_{s}\right), g_{n}(x) \neq 0$ for $v$-almost every $x \in \mathbf{K}$ and $\left\|g_{n}\right\|=1$ for each $n$, for which converges $\prod_{n=1}^{\infty} \beta_{n}>0$ for each $y \in J$ and such that $\prod_{n=1}^{m} g_{n}\left(x_{n}\right) w^{\prime}\left(d x_{n} / a_{n}\right)=$ : $\nu_{L_{n}}\left(d x^{n}\right)$ satisfies $\left[24,25,26\right.$, conditions of Lemma 2.3], where $\beta_{n}:=\left\|\rho_{n}\right\|_{\phi_{n}}, 0 \neq a_{n} \in$ K for each $n \in \mathbb{N}, \rho_{n}(x):=\mu_{n}(d x) / \nu_{n}(d x), \phi_{n}(x):=N_{\lambda_{n}}(x), \lambda_{n}(d x):=g_{n}(x) w^{\prime}\left(d x / a_{n}\right)$, then use $\left[24,25,26\right.$, Theorem 3.5] for the measure $\nu_{n}(d x):=g_{n}(x) w^{\prime}\left(d x / a_{n}\right)$ and $\mu_{n}(d x)$ $:=v_{n}\left(-y_{n}+d x\right), x^{n}:=\left(x_{1}, \ldots, x_{n}\right), x_{1}, \ldots, x_{n} \in \mathbf{K}$ for each $n \in \mathbb{N}$. The family of such sequences of functions $\left\{g_{n}: n \in \mathbb{N}\right\}$ has the cardinality card $\left(\mathbf{K}_{\mathbf{s}}\right)^{\mathrm{c}}$, since in $L(\nu)$ the subspace of step functions is dense and $\operatorname{card}(\mathrm{B} \operatorname{co}(X))=\mathrm{c}$. The family of all $\left\{g_{n}: n\right\}$ satisfying conditions above for $J$ also satisfies such conditions for $J^{\prime}$. From which the latter statement of this theorem follows.

Theorem 5.8. Let $X$ be a Banach space of separable type over a locally compact nonArchimedean field $\mathbf{K}$ and $J$ be a dense proper $\mathbf{K}$-linear subspace in $X$ such that the embedding operator $T: J \hookrightarrow X$ is compact and nondegenerate, $\operatorname{ker}(T)=\{0\}, b \in \mathbb{C}$. Then a set $\mathscr{P}_{b}(X, J)$ of probability $\mathbf{K}_{\mathbf{s}}$-valued measures $\mu$ on $\mathrm{B} \operatorname{co}(X)$ quasi-invariant and pseudodifferentiable of order $b$ relative to $J$ is of cardinality $\operatorname{card}\left(\mathbf{K}_{\mathrm{s}}\right)^{\mathrm{c}}$. If $J^{\prime}, J^{\prime} \subset J$, is also a dense $\mathbf{K}$-linear subspace in $X$, then $\mathscr{P}_{b}\left(X, J^{\prime}\right) \supset \mathscr{P}_{b}(X, J)$.

Proof. As in [24, Theorem 4.5] choose for $T$ an orthonormal base in $X$ in which $T$ is diagonal and $X$ is isomorphic with $c_{0}$ over $\mathrm{K}$ such that in its standard base $\left\{e_{j}: j \in \mathbb{N}\right\}$ the operator $T$ is characterized by $T e_{j}=a_{j} e_{j}, 0 \neq a_{j} \in \mathbf{K}$ for each $j \in \mathbb{N}, \lim _{j \rightarrow \infty} a_{j}=0$. Take $g_{n}$ from Theorem 5.7, where $g_{n} \in L\left(\mathbf{K}, B f(\mathbf{K}), w^{\prime}\left(d x / a_{n}\right), \mathbf{K}_{\mathbf{s}}\right)$, satisfy conditions there and such that there exists $\lim _{m \rightarrow \infty} \operatorname{PD}\left(b, \prod_{n=1}^{m} g_{n}(x z)\right) \in L(X, \operatorname{Bco}(X), v, \mathbf{F})$ by the variable $x$ for each $z \in J$, where $x \in \mathbf{K}, \mathbf{K}_{\mathbf{s}} \cup \mathbb{C}_{\mathbf{s}} \subset \mathbf{F}$, and $\mathbf{F}$ is a non-Archimedean field. Evidently, $\mathscr{P}_{b}(X, J) \subset \mathcal{M}(X, J)$. The family of such sequences of functions $\left\{g_{n}: n \in \mathbb{N}\right\}$ has the cardinality $\operatorname{card}\left(\mathbf{K}_{\mathbf{s}}\right)^{\mathrm{c}}$, since in $L(\nu)$ the subspace of step functions is dense and the condition of pseudodifferentiability is the integral convergence condition (see $[24,25,26$, Sections 4.1 and 4.2]).

\section{References}

[1] S. Albeverio and W. Karwowski, Diffusion on p-adic numbers, Gaussian Random Fields (Nagoya, 1990) (K. Itô and T. Hida, eds.), Ser. Probab. Statist., vol. 1, World Scientific, New Jersey, 1991, pp. 86-99.

[2] I. Ya. Aref'eva, B. G. Dragović, and I. V. Volovich, On the p-adic summability of the anharmonic oscillator, Phys. Lett. B 200 (1988), no. 4, 512-514.

[3] C. Castro, Fractal strings as an alternative justification for El Naschie's cantorian spacetime and the fine structure constants, Chaos Solitons Fractals 14 (2002), no. 9, 1341-1351.

[4] Yu. L. Dalecky and S. V. Fomin, Measures and Differential Equations in Infinite-Dimensional Space, Mathematics and Its Applications (Soviet Series), vol. 76, Kluwer Academic, Dordrecht, 1991.

[5] B. Diarra, Ultraproduits ultramétriques de corps valués [Ultrametric ultraproducts of valued fields], Ann. Sci. Univ. Clermont-Ferrand II Math. 22 (1984), 1-37. 
[6] G. S. Djordjević and B. Dragovich, p-adic and adelic harmonic oscillator with a time-dependent frequency, Theoret. and Math. Phys. 124 (2000), no. 2, 1059-1067 (Russian).

[7] R. Engelking, General Topology, Mir, Moscow, 1986.

[8] A. Escassut, Analytic Elements in p-Adic Analysis, World Scientific, New Jersey, 1995.

[9] I. M. Gel'fand and N. Ya. Vilenkin, Generalized Functions, No. 4: Some Applications of Harmonic Analysis. Equipped Hilbert Spaces, Gosudarstv. Izdat. Fiz.-Mat. Lit., Moscow, 1961.

[10] Y. Jang, Non-Archimedean Quantum Mechanics, Tohoku Mathematical Publications, vol. 10, Tohoku University Mathematical Institute, Sendai, 1998.

[11] A. Yu. Khrennikov, Mathematical methods in non-Archimedean physics, Russian Math. Surveys 45 (1990), no. 4, 87-125 (Russian).

[12] - Generalized functions and Gaussian path integrals over non-Archimedean function spaces, Izv. Akad. Nauk SSSR Ser. Mat. 55 (1991), no. 4, 780-814 (Russian).

[13] Interpretations of Probability, VSP, Utrecht, 1999.

[14] N. Koblitz, p-Adic Numbers, p-Adic Analysis, and Zeta-Functions, Graduate Texts in Mathematics, vol. 58, Springer, New York, 1977.

[15] S. V. Lüdkovsky, Measures on groups of diffeomorphisms of non-Archimedean manifolds, representations of groups and their applications, Theoret. and Math. Phys. 119 (1999), no. 3, 698-711 (Russian).

[16] Non-Archimedean polyhedral expansions of ultra-uniform spaces, Russian Math. Surveys 54 (1999), no. 5, 1029-1030 (Russian), (detailed version: Los Alamos National Laboratory, USA. preprint http://arxiv.org/abs/math.AT/0005205, 39 pages, May 2000).

[17] _ Properties of quasi-invariant measures on topological groups and associated algebras, Ann. Math. Blaise Pascal 6 (1999), no. 1, 33-45.

[18] Non-Archimedean polyhedral decompositions of ultra-uniform spaces, Fundam. Prikl. Mat. 6 (2000), no. 2, 455-475 (Russian).

[19] Quasi-invariant measures on non-Archimedean groups and semigroups of loops and paths, their representations. I, Ann. Math. Blaise Pascal 7 (2000), no. 2, 19-53.

[20] Quasi-invariant measures on non-Archimedean groups and semigroups of loops and paths, their representations. II, Ann. Math. Blaise Pascal 7 (2000), no. 2, 55-80.

[21] Stochastic processes on groups of diffeomorphisms and loops of real, complex, and nonArchimedean manifolds, Fundam. Prikl. Mat. 7 (2001), no. 4, 1091-1105 (Russian).

[22] _ Quasi-invariant and pseudo-differentiable real-valued measures on non-Archimedean Banach spaces, Anal. Math. 28 (2002), no. 4, 287-316 (Russian).

[23] Quasi-invariant and pseudo-differentiable measures on non-Archimedean Banach spaces, Russian Math. Surveys 58 (2003), no. 2, 377-378 (Russian).

[24]_, Quasi-invariant and pseudo-differentiable measures with values in non-Archimedean fields on a non-Archimedean Banach space, J. Math. Sci. (New York) 122 (2004), no. 1, 29492983.

[25] ( Quasi-invariant and pseudo-differentiable measures on a non-Archimedean Banach space. I. Real-valued measures, Los Alamos preprints, 2001, http://arxiv.org/abs/ math.GM/0106169.

[26] _ Quasi-invariant and pseudo-differentiable measures on a non-Archimedean Banach space. II. Measures with values in non-Archimedean fields, Los Alamos preprints, 2001, http://arxiv.org/abs/math.GM/0106170.

[27] S. V. Lüdkovsky and A. Yu. Khrennikov, Stochastic processes on non-Archimedean spaces with values in non-Archimedean fields, Markov Process. Related Fields 9 (2003), no. 1, 131-162.

[28] L. Narici and E. Beckenstein, Topological Vector Spaces, Monographs and Textbooks in Pure and Applied Mathematics, vol. 95, Marcel Dekker, New York, 1985.

[29] W. H. Schikhof, Ultrametric Calculus. An Introduction to p-Adic Analysis, Cambridge Studies in Advanced Mathematics, vol. 4, Cambridge University Press, Cambridge, 1984. 
[30] A. C. M. van Rooij, Non-Archimedean Functional Analysis, Monographs and Textbooks in Pure and Applied Math., vol. 51, Marcel Dekker, New York, 1978.

[31] V. S. Vladimirov and I. V. Volovich, p-adic quantum mechanics, Comm. Math. Phys. 123 (1989), no. 4, 659-676.

[32] V. S. Vladimirov, I. V. Volovich, and E. I. Zelenov, p-Adic Analysis and Mathematical Physics, Nauka, Moscow, 1994.

[33] A. Weil, Basic Number Theory, Springer, Berlin, 1973.

S. V. Lüdkovsky: Chair of Applied Mathematics, Moscow State Technical University MIREA, 78 Vernadsky Avenue, Moscow 119454, Russia

E-mail addresses: sludkovs@vub.ac.be; sludkowski@mail.ru 


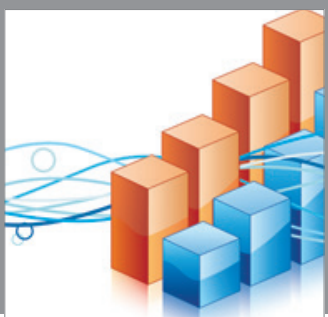

Advances in

Operations Research

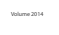

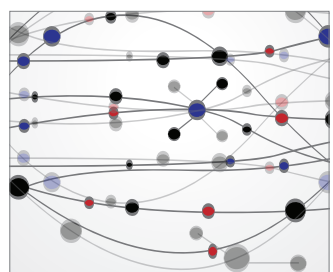

\section{The Scientific} World Journal
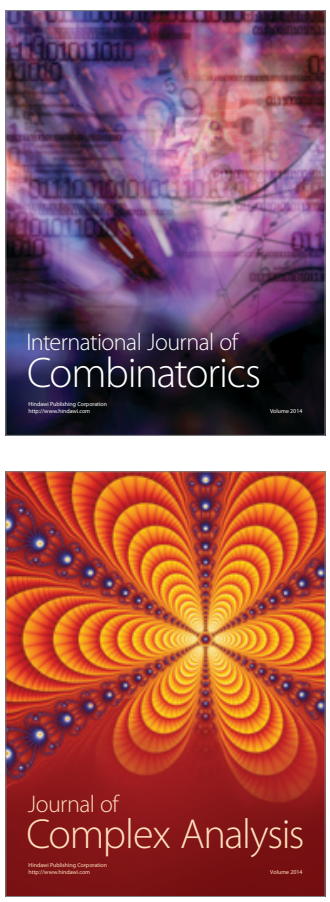

International Journal of

Mathematics and

Mathematical

Sciences
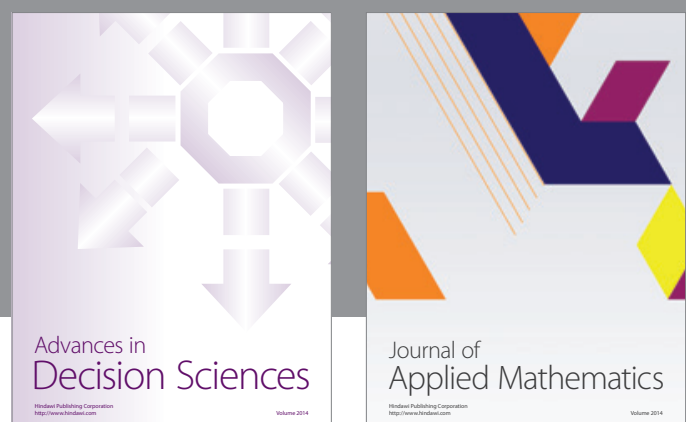

Journal of

Applied Mathematics
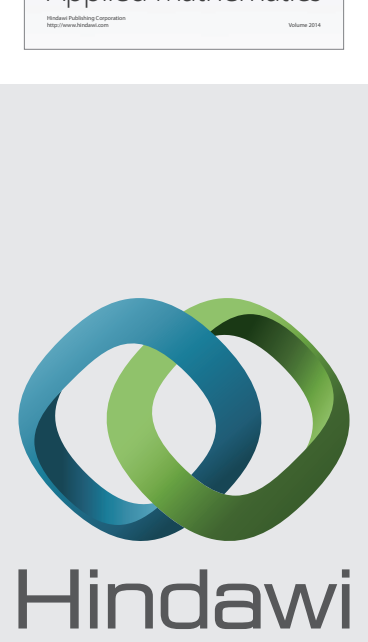

Submit your manuscripts at http://www.hindawi.com
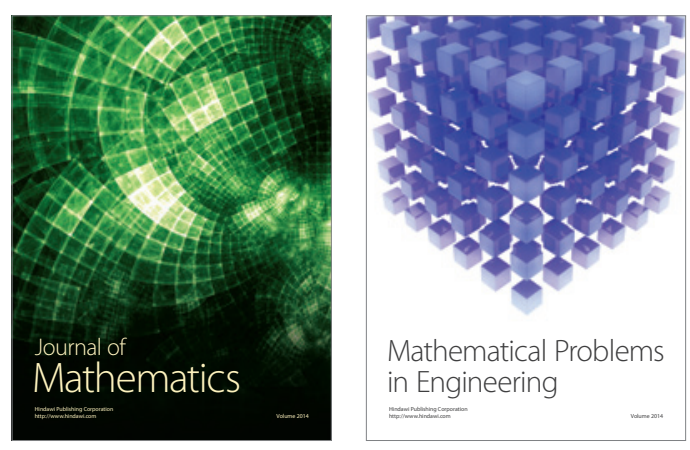

Mathematical Problems in Engineering
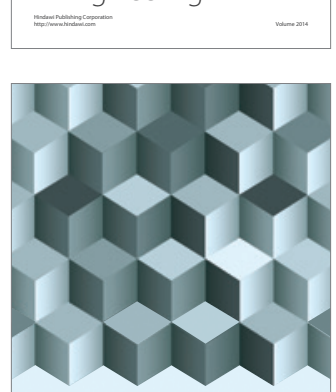

Journal of

Function Spaces
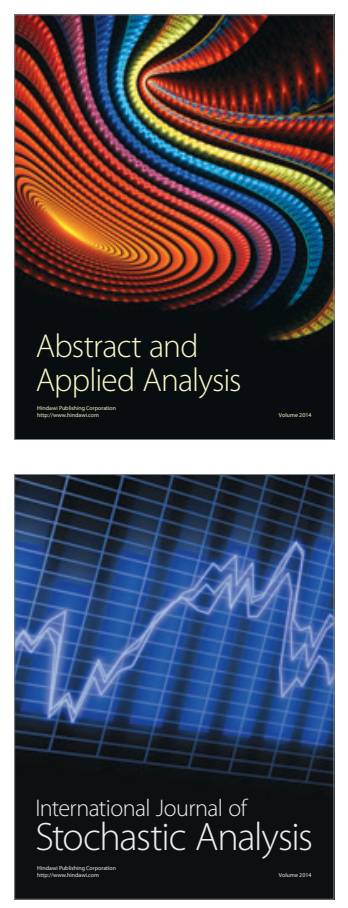

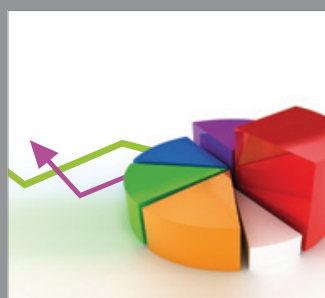

ournal of

Probability and Statistics

Promensencen
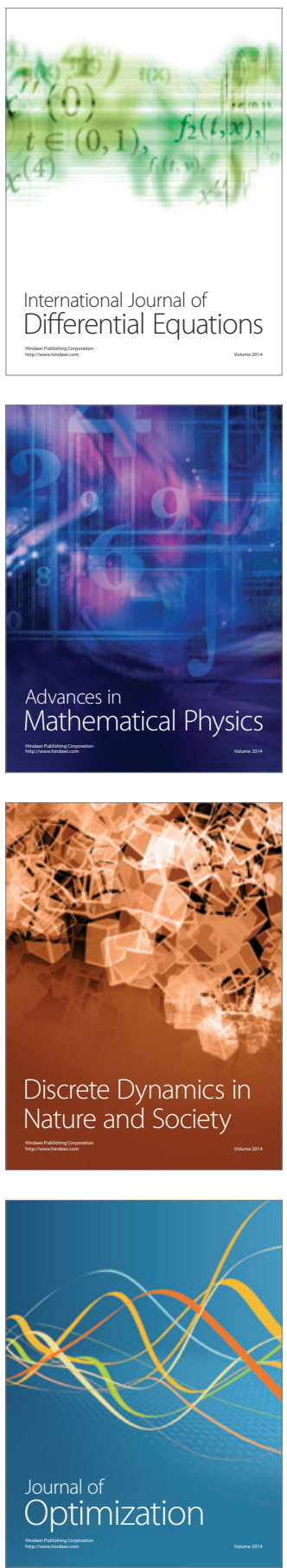